Argonne

ANL-18/27

\title{
Analysis of Achievable Rates of Communication
}

Transmission of Information by Acoustic Communication along Metal Pathways in Nuclear Facilities

Nuclear Science and Engineering Division 


\title{
About Argonne National Laboratory
}

Argonne is a U.S. Department of Energy laboratory managed by UChicago Argonne, LLC under contract DE-AC02-06CH11357. The Laboratory's main facility is outside Chicago, at 9700 South Cass Avenue, Argonne, Illinois 60439. For information about Argonne and its pioneering science and technology programs, see www.anl.gov.

\section{Document availability}

Online Access: U.S. Department of Energy (DOE) reports produced after 1991 and a growing number of pre-1991 documents are available free at OSTI.GOV (http://www.osti.gov/), a service of the U.S. Dept. of Energy's Office of Scientific and Technical Information

\author{
Reports not in digital format may be purchased by the public from the \\ National Technical Information Service (NTIS): \\ U.S. Department of Commerce \\ National Technical Information Service \\ 5301 Shawnee Rd \\ Alexandria, VA 22312 \\ www.ntis.gov \\ Phone: (800) 553-NTIS (6847) or (703) 605-6000 \\ Fax: (703) 605-6900 \\ Email: orders@ntis.gov
}

\section{Reports not in digital format are available to DOE and DOE contractors from the Office of Scientific and Technical Information (OSTI):}

U.S. Department of Energy

Office of Scientific and Technical Information

P.O. Box 62

Oak Ridge, TN 37831-0062

www.osti.gov

Phone: (865) 576-8401

Fax: (865) 576-5728

Email: reports@osti.gov

This report was prepared as an account of work sponsored by an agency of the United States Government. Neither the United States Government nor any agency thereof, nor UChicago Argonne, LLC, nor any of their employees or officers, makes any warranty, express or implied, or assumes any legal liability or responsibility for the accuracy, completeness, or usefulness of any information, apparatus, product, or process disclosed, or represents that its use would not infringe privately owned rights. Reference herein to any specific commercial product, process, or service by trade name, trademark, manufacturer, or otherwise, does not necessarily constitute or imply its endorsement, recommendation, or favoring by the United States Government or any agency thereof. The views and opinions of document authors expressed herein do not necessarily state or reflect those of the United States Government or any agency thereof, Argonne National Laboratory, or UChicago Argonne, LLC. 


\section{Analysis of Achievable Rates of Communication}

Transmission of Information by Acoustic Communication along Metal Pathways in Nuclear Facilities

prepared by

Alexander Heifetz ${ }^{1}$, Xin Huang ${ }^{1,2}$, Dmitry Shribak ${ }^{1,3}$, Sasan Bakhtiari ${ }^{1}$, Jafar Saniie ${ }^{2}$, and Richard B. Vilim ${ }^{1}$

${ }^{1}$ Nuclear Science Engineering Division, Argonne National Laboratory

${ }^{2}$ Department of Electrical and Computer Engineering, Illinois Institute of Technology, Chicago, IL

${ }^{3}$ Department of Physics, University of Chicago, Chicago, IL 


\section{Table of Contents}

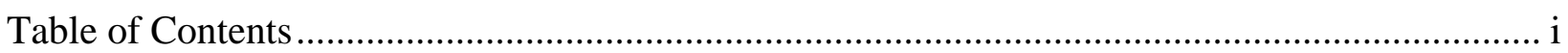

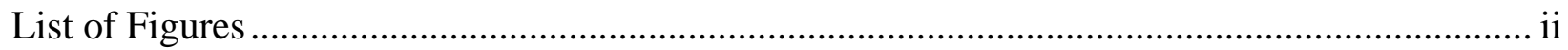

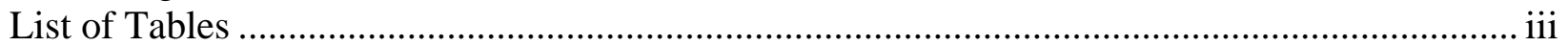

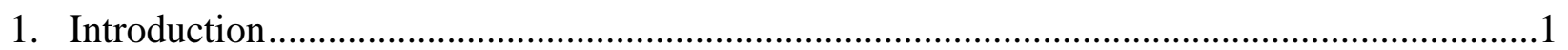

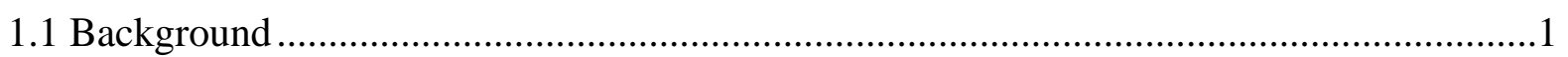

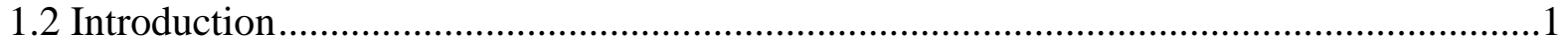

2. Description of Software Defined Radio Protocol for Acoustic Communication on Pipes........3

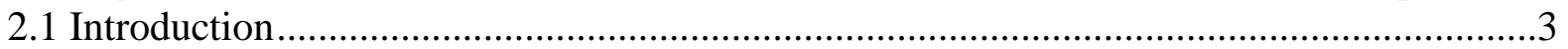

2.2 Modulation Techniques ...............................................................................................

2.3 GNURadio Communication Protocol Implementation..................................................4

2.4 Communication Protocol Testing and Analysis ......................................................11

2.5 Comparison of Communication Protocols Performance ................................................21

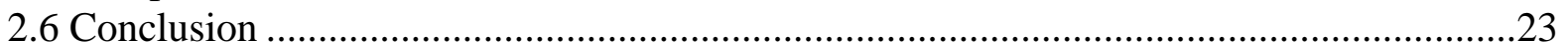

3. Demonstration of Information Transmission with Acoustic Communication System ............24

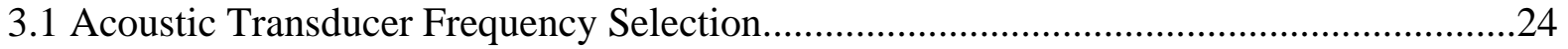

3.2 Wedge Selection for PZT Refracted Shear Wave.....................................................26

3.3 Demonstration Data Transmission with PZT-based Channel...........................................28

3.4 Preliminary Evaluation of Data Transmission with EMAT-based Channel.....................29

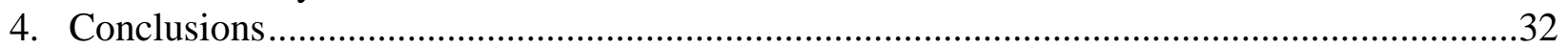

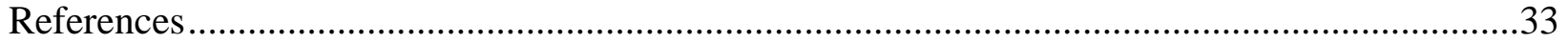




\section{List of Figures}

Figure 1 - Proposed acoustic communication system at a nuclear facility ............................... 1

Figure 2 - Schematics of shift keying modulation techniques. .............................................. 3

Figure 3 - ASK acosutic communication protocol flow chart created with GNURadio............... 6

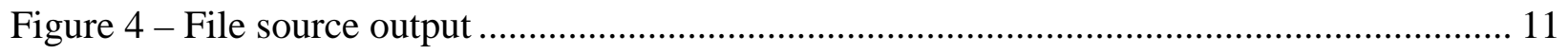

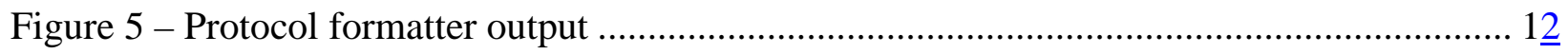

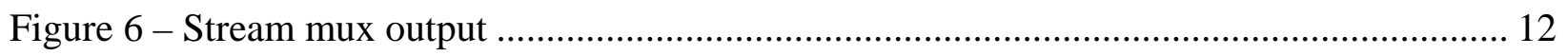

Figure 7 - Bit value output ...................................................................................... $1 \underline{3}$

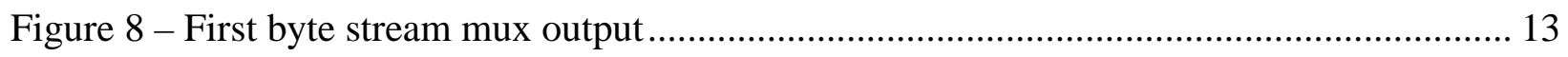

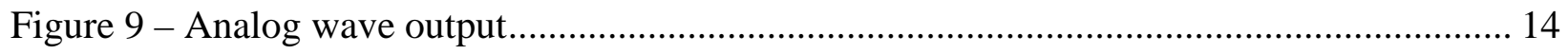

Figure 10 - FFT spectrum of analog wave output ............................................................ 14

Figure 11 - Transmitted wave on pipe .............................................................................. 15

Figure 12 - FFT spectrum of transmitted wave on pipe .................................................... 15

Figure 13 - Received wave on pipe before demodulation ...................................................... 16

Figure 14 - FFT spectrum of received wave on pipe before demodulation ............................... 16

Figure 15 - First byte in received signal before demodulation .............................................. 17

Figure 16 - Received demodulated signal with Red Pitaya board ........................................... 17

Figure 17 - FFT spectrum of demodulated signal received with Red Pitaya board ................... 18

Figure 18 - Zoomed-in received signal from Red Pitaya board ............................................ 18

Figure 19 - Signal amplification with automatic gain control ............................................... 19

Figure 20 - Data of thershould block after demodulation ...................................................... 20

Figure 21 - Data recovered after decimation ................................................................ 20

Figure 22 - Data recovered with correlated access code ..................................................... 21

Figure 23 - Data from file sink input ......................................................................... 21

Figure 24 - BER vs. SNR for ASK, ASK with CC, PSK, and PSK with CC schemes ............. 22

Figure 25 - BER vs. carrier frequency for ASK and ASK with CC schemes .......................... 25

Figure 26 - Informatio-carrying waveform in time and frequency domain .............................. 24

Figure 27 - Amplitude-modulated carrier waveform in time and frequency domain ................ 24

Figure 28 - Received signal multiplied by reference in time and frequency domain ................ 25

Figure 29 - Communication channel based on refracted shear wave in stainless steel plate and pipe

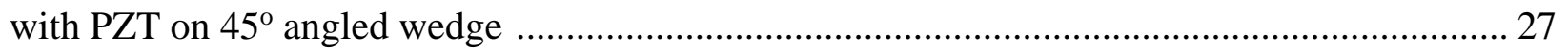

Figure 30 - Time domain signals on plate and pipe ........................................................ 27

Figure 31 - Schematics of communication system setup for image transmission on a pipe ....... 28

Figure 32 - Transmission of text file .......................................................................... 29

Figure 33 - Schematics of EMAT-based communication channel on a plate ........................... 29

Figure 34 - Signals reflections and interferences in EMAT-based channel Error! Bookmark not defined. 0 


\section{List of Tables}

Table 1 - Example of header file .......................................................................................... 4

Table 2 - ASCII to number conversion table ...................................................................... 5

Table 3 - Functionalities of blocks before signal modulation steps......................................... 7

Table 4 - Functionalities of blocks during signal modulation steps ......................................... 8

Table 5 - Functionalities of blcoks after signal modulation steps......................................... 10 


\section{Introduction}

\subsection{Background}

Nuclear Science Engineering Division at Argonne has been developing a new method of transmitting information via elastic waves on pipes in a nuclear facility. Compared to traditional RF communications, acoustic communications allows for signal transmission across physical barriers, and better information security due to spatial confinement of the signal. Such communication system would take advantage of the existing piping infrastructure to transmit information in and out of containment building, as shown in Figure 1. Data of interest consists of sensor readings, sound, and images. This approach would provide a communication channel to the containment building containment building under the conditions of total power outage following a severe accident.

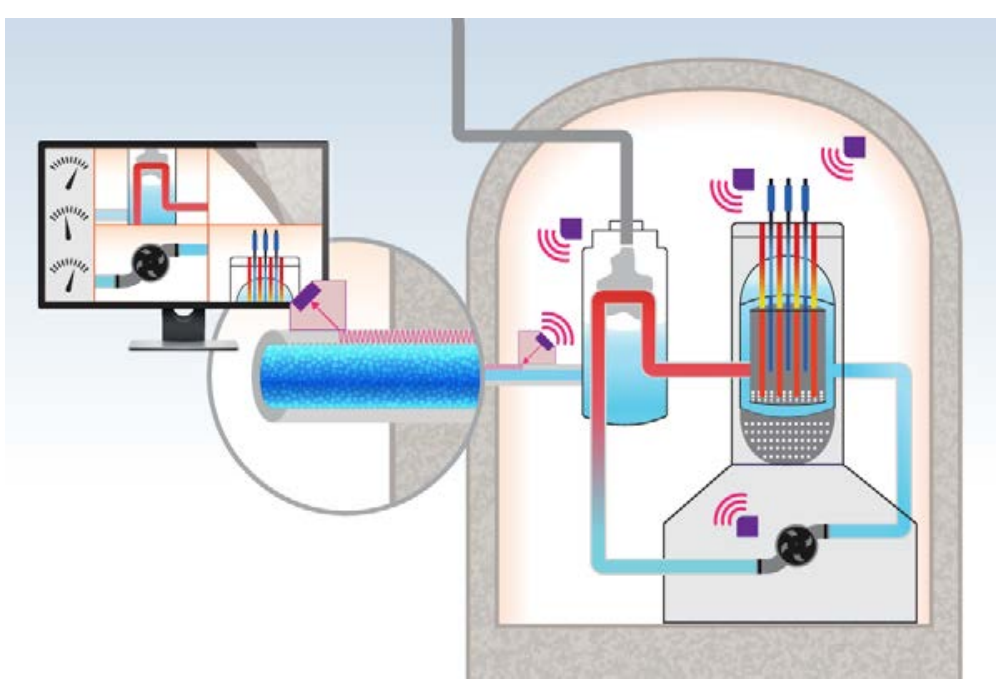

Figure 1 - Proposed acoustic communication system at a nuclear facility would transmit information on steel pipes already in place for nuclear reactor operation (Argonne 2018).

\subsection{Introduction}

In this project, we are developing a wireless communication system for a nuclear facility, in which information is carried with elastic waves propagating on metallic pipes. Our project combines the fields of acoustics with communication technology. Because of limited bandwidth of ultrasonic transducers, on-off-keying (OOK) digital communication protocol was chosen for information transmission over an acoustic channel. Current work on the project is focused on investigating communication channel capacity (bit rate or duty cycle of the analog signal amplitude waveform), reliability (bit error rate) and energy efficiency (energy consumption per bit) of OOK. Design considerations of the OOK communication protocol are intimately connected to physical characteristics of the communication channel. 
In general, communication data rate is proportional to carrier frequency. However, signal attenuation in the channel due to absorption is proportional to carrier frequency as well, so that carrier frequency cannot be increased indefinitely. Prior work demonstrated that pulse energy fades below detection threshold if the pulse temporal duration is smaller than some limit. Other factors adversely influencing communication bit rate studied more recently include mixture of modes and echoes. Longitudinal and shear waves have different propagation velocities in stainless steel, so that excitation of both modes can lead to bit errors. Excitation of shear waves only can be achieved with EMAT (electromagnetic acoustic transducers). Refracted shear waves can be excited with PZT (piezo-electric transducers) mounted on angled wedge, with an angle larger than the first critical angle. Echoes occur due to reflections of acoustic waves at discontinuities, such as pipe ends. Echoes are stronger at lower frequency, which suffers less attenuation from absorption losses in the channel. In addition, only round-trip echoes, re-scattered in the direction of the original signal, couple into the angled-wedge mounted PZT receiver. On the other hand, EMAT receiver is omnidirectional, and picks up echoes from every scattered in any direction towards the receiver. Thus, longer distance of echo propagation before coupling into receiver makes PZT-based channel more immune to echoes. Some aspects of the communication protocol affecting data bit rate include length of time required to achieve optimal performance with active control gain, and received analog signal decimation procedure.

This report describes implementation of amplitude shift keying (ASK) communication protocol using GNURadio software environment, and evaluation performance of communication channels based on PZT and EMAT transducers. Main achievements thus far include demonstration of transmission of sound and text files with PZT and EMAT across six-foot long nuclear grade stainless steel pipe, and demonstration of image transmission with PZT over the pipe. In the former example, $32 \mathrm{~KB}$ image was transmitted at data rate of $2 \mathrm{~KB} / \mathrm{s}$. Efforts are currently under way to further enhance data transmission rate. 


\section{Description of Software Defined Radio Protocol for Acoustic Communication on Pipes}

\subsection{Introduction}

Data of interest for transmission on nuclear reactor pipes includes sensor readings, voice, and images. Transmission of images is most challenging because corruption of the header string might render the reconstructed image unreadable. We used the Software Defined Radio (SDR) tool GNURadio, which allowed us to build a communication protocol to transmit data effectively. We created a Python tool to compare different modulation and front error correction techniques used to transferring header files. The goal of this task was to successfully transmit the header file of a .ppm (Portable Pixel Map) image because any corruption in this data renders the image useless.

For the physical transmission, we employed two ultrasonic PZT's on a six foot-long stainless steel pipe spaced at about five feet apart. The transducers were originally designed for nondestructive testing. A digital wave is generated by the GNURadio program, which is next converted into an analog wave by Red Pitaya electronic boards. This analog wave is sent to the PZT through an amplifier, which converts the electronic wave into an acoustic wave by vibrating PZT crystal at a certain frequency. This vibration passes through the pipe in the form of a shear mechanical wave and vibrates the transducer on the other end of the pipe, generating an electrical wave, which is converted back into a digital wave by Red Pitaya.

\subsection{Modulation Techniques}

Digital modulation is the process sending a binary signal inside of a different signal that can be easily transmitted over a physical channel. In our case, we chose to employ binary Amplitude Shift Keying (ASK) and binary Phase Shift Keying (PSK). Schematics of shift keying modulation techniques are displayed in Figure 2.

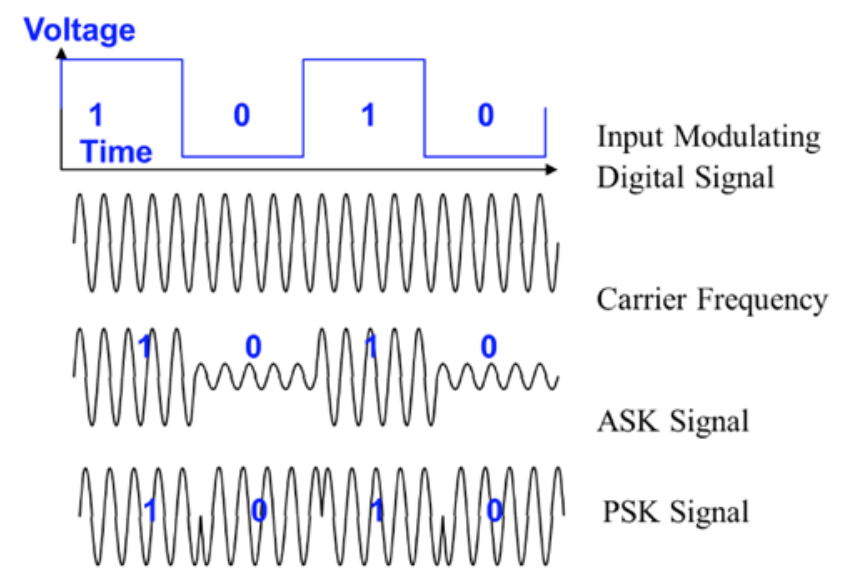

Figure 2 - Schematics of shift keying modulation techniques 
In ASK, a 1 is represented as a high amplitude, and a 0 is represented by a low amplitude. In PSK, a 1 is represented by a 180 degree phase shift on the carrier frequency, and a 0 is represented by a 0 degree phase shift on the carrier frequency. The binary waveform is multiplied onto a carrier frequency, produced by the Red Pitaya, and transmitted through the pipe. On the receiving end, the signal is demodulated by multiplying again by the carrier frequency and passing the information through a low pass filter to recover the original signal. These are the most simple forms of modulation available, and while more elaborate forms of Shift Keying exist that allow for more information to be encoded per key, we chose this technique due to the fact that the constellation symbols are maximally spread out, reducing the impact of noise on the channel compared to a setup that encodes more than one bit per symbol.

\subsection{GNURadio Communication Protocol Implementation}

In communication protocol implemented in SDR, image data has to be processed in order to be successfully transmitted and recovered. As noted above, only a binary stream of information can be sent through the pipe. However, image data has a non-binary data structure and for that we have chosen to use a Portable Pixel Map (PPM) file as our primary image data structure for transmission.

\section{Table 1. Example of header file}

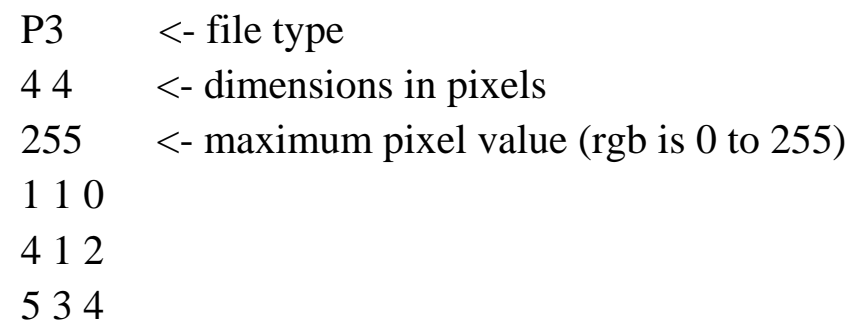

The PPM file type stores the image dimensions in the first three lines and the rest is the image itself as a matrix after that in the form of ASCII character. We will refer to these three lines as the "image header" and the rest as the "image payload". The image header is critical to successful recovery of the image, as if there are any errors within the file, the computer will not be able to read the data effectively. The image payload, on the other hand, can contain more errors as a single error is less likely to make the file unreadable.

To transmit this information, data structure has to be converted into binary, sent, and reassembled back into its original form. To accomplish this task, we first disassemble the file into a stream of ASCII characters, in the form of bytes ( 1 byte $=8$ bits). A table of ASCII values is shown below: 
Table 2. ASCII to number conversion table

\begin{tabular}{|c|c|c|c|c|c|}
\hline ASCII & Character A & ASCII & Character & ASCII & Character \\
\hline value & & value & & valu & \\
\hline 000 & -0 & 043 & + & 086 & $\mathbf{v}$ \\
\hline 001 & $-A$ & 044 & , & 087 & H \\
\hline 002 & ${ }^{-} \mathbf{B}$ & 045 & - & 088 & $\mathbf{X}$ \\
\hline 003 & ${ }^{-C}$ & 046 & . & 089 & $\mathbf{Y}$ \\
\hline 004 & $-D$ & 047 & $f$ & 090 & $\mathbf{Z}$ \\
\hline 005 & $-E$ & 048 & 0 & 091 & {[} \\
\hline 006 & $-F$ & 049 & 1 & 092 & 1 \\
\hline 007 & ${ }^{-G}$ & 050 & 2 & 093 & ] \\
\hline 008 & ${ }^{-} \mathrm{H}$ & 051 & 3 & 094 & - \\
\hline 009 & $-I$ & 052 & 4 & 095 & - \\
\hline 010 & $-\mathrm{J}$ & 053 & 5 & 096 & $r$ \\
\hline 011 & ${ }^{-} \mathbf{K}$ & 054 & 6 & 097 & $\mathbf{a}$ \\
\hline 012 & ${ }^{-} L$ & 055 & 7 & 098 & $\mathbf{b}$ \\
\hline 013 & $-\mathrm{H}$ & 056 & $\mathbf{8}$ & 099 & $\mathbf{c}$ \\
\hline 014 & $-\mathbf{N}$ & 057 & 9 & 100 & d \\
\hline 015 & ס & 158 & : & 101 & $\mathbf{e}$ \\
\hline 016 & $-P$ & 059 & ; & 102 & $f$ \\
\hline 017 & $-Q$ & 060 & $<$ & 103 & $\mathbf{g}$ \\
\hline 018 & $-\mathrm{R}$ & 061 & $=$ & 104 & $\mathbf{h}$ \\
\hline 019 & -5 & 062 & $>$ & 105 & $i$ \\
\hline 020 & $-T$ & 063 & $?$ & 106 & j \\
\hline 021 & ${ }^{-} \mathrm{U}$ & 064 & a & 107 & $\mathbf{k}$ \\
\hline 022 & $-\mathrm{V}$ & 065 & A & 108 & 1 \\
\hline 023 & $-\mathrm{H}$ & 066 & B & 109 & $\mathbf{m}$ \\
\hline 024 & $-x$ & 067 & C & 110 & $\mathbf{n}$ \\
\hline 025 & ${ }^{-} \mathbf{Y}$ & 068 & D & 111 & 0 \\
\hline 026 & $-z$ & 069 & $\mathbf{E}$ & 112 & $\mathbf{p}$ \\
\hline 027 & -[ & 070 & $\mathbf{F}$ & 113 & $\mathrm{q}$ \\
\hline 028 & -1 & 071 & G & 114 & $\mathbf{r}$ \\
\hline 029 & $-]$ & 072 & $\mathbf{H}$ & 115 & $\mathbf{s}$ \\
\hline 030 & $m$ & 073 & $\mathbf{I}$ & 116 & $\mathbf{t}$ \\
\hline 031 & $=$ & 074 & $\mathbf{J}$ & 117 & $\mathbf{u}$ \\
\hline 032 & [space] & 075 & $\mathbf{K}$ & 118 & $\nabla$ \\
\hline 033 & $!$ & 076 & $\mathbf{L}$ & 119 & $\nabla$ \\
\hline 034 & $"$ & 077 & M & 120 & $\mathbf{x}$ \\
\hline 035 & $\#$ & 078 & $\mathbf{N}$ & 121 & $y$ \\
\hline 036 & $\$$ & 079 & D & 122 & $\mathbf{z}$ \\
\hline 037 & $\%$ & 080 & $\mathbf{P}$ & 123 & {[} \\
\hline 038 & $z$ & 081 & Q & 124 & I \\
\hline 039 & , & 082 & $\mathbf{R}$ & 125 & \} \\
\hline 040 & ( & 083 & $\mathrm{~S}$ & 126 & - \\
\hline 041 & ) & 084 & $\mathbf{T}$ & 127 & DEL \\
\hline 042 & $*$ & 085 & $\mathbf{U}$ & & \\
\hline
\end{tabular}

These characters are then converted into a bit stream, a packet header is attached and is passed through the modulation methods mentioned earlier. After being demodulated, the bit stream is parse to remove the packet header, the bits are converted into their original bytes, and the file is reassembled. When error correction is added, after the bytes are converted into bits they are multiplied by a convolution matrix, and once received, the bits are deconvoluted by an inverse 
matrix. Overall, the protocol of the communication system was the primary focus of this project as reducing the number of errors in transmitting ensures that the communication system is reliable. GNURadio environment was used to develop and implement the communication protocol. Figure 3 below displays a flowgraph generated by GNURadio for ASK modulation with no convolutional code. Descriptions of various blocks functionalities are provided in tables below.

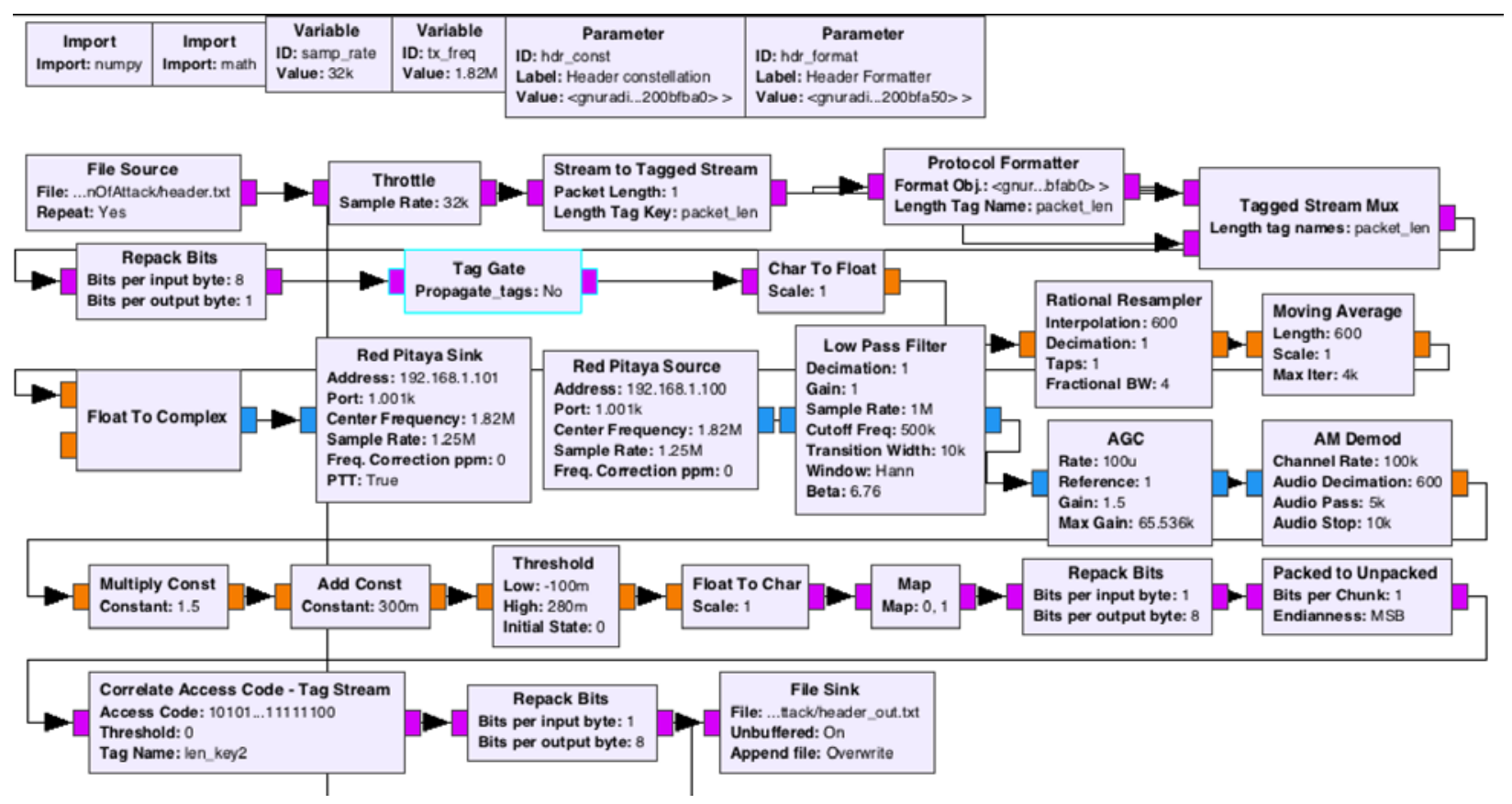

Figure 3 - ASK acoustic communication protocol flow chart created by GNURadio

Explanations of blocks functionalities before modulation, during modulation, and after modulation steps are provided in Tables 3, 4 and 5, respectively. 
Table 3. Functionalities of blocks before signal modulation steps

\begin{tabular}{|c|c|c|c|c|c|}
\hline Block & Input & Output & Parameters & Actions & Purpose \\
\hline File Source & $\begin{array}{l}\text { Text file } \\
\text { containing } \\
\text { information to } \\
\text { be sent }\end{array}$ & $\begin{array}{l}\text { Characters } \\
\text { in the file in } \\
\text { form of } \\
\text { continuous } \\
\text { stream of } \\
\text { bytes }\end{array}$ & $\begin{array}{l}\text { Output type: Whether } \\
\text { the file is a stream of } \\
\text { bytes, floats, ints, etc; } \\
\text { Repeat: Whether the } \\
\text { file will be infinitely } \\
\text { repeated; }\end{array}$ & & $\begin{array}{l}\text { Opens the } \\
\text { needed data } \\
\text { file }\end{array}$ \\
\hline Throttle & - & - & $\begin{array}{l}\text { Sample Rate: Number } \\
\text { of samples per second }\end{array}$ & $\begin{array}{l}\text { Limits the } \\
\text { flow of } \\
\text { information } \\
\text { to } 32 \mathrm{k} \\
\text { samples/sec }\end{array}$ & $\begin{array}{l}\text { Makes sure } \\
\text { computer } \\
\text { doesn't } \\
\text { overheat }\end{array}$ \\
\hline $\begin{array}{l}\text { Stream to } \\
\text { tagged } \\
\text { stream }\end{array}$ & $\begin{array}{l}\text { Continuous } \\
\text { stream of } \\
\text { characters }\end{array}$ & $\begin{array}{l}\text { Continuous } \\
\text { stream of } \\
\text { characters } \\
\text { with tagged }\end{array}$ & $\begin{array}{l}\text { Packet Length: Controls } \\
\text { how much data stored is } \\
\text { per packet }\end{array}$ & & $\begin{array}{l}\text { Puts a marker } \\
\text { on the start of } \\
\text { a packet for } \\
\text { the header } \\
\text { generator to } \\
\text { attach to }\end{array}$ \\
\hline $\begin{array}{l}\text { Protocol } \\
\text { Formatter }\end{array}$ & $\begin{array}{l}\text { Continuous } \\
\text { tagged stream } \\
\text { of characters }\end{array}$ & $\begin{array}{l}\text { String of } \\
\text { header } \\
\text { characters }\end{array}$ & $\begin{array}{l}\text { Formater Obj: Sets the } \\
\text { protocol formatter's key } \\
\text { parameters: Preamble } \\
\text { and Access Code, } \\
\text { which are basically } \\
\text { what the correlate } \\
\text { access code will look } \\
\text { for when assessing } \\
\text { what is a packet }\end{array}$ & $\begin{array}{l}\text { Creates a } \\
\text { header for } \\
\text { the packet }\end{array}$ & \\
\hline $\begin{array}{l}\text { Tagged } \\
\text { Stream Mux }\end{array}$ & $\begin{array}{l}\text { Two tagged } \\
\text { streams }\end{array}$ & $\begin{array}{l}\text { One tagged } \\
\text { stream }\end{array}$ & $\begin{array}{l}\text { Length tag names: } \\
\text { Input the length of the } \\
\text { data stream to which to } \\
\text { attach the header }\end{array}$ & $\begin{array}{l}\text { Combines } \\
\text { the two } \\
\text { data } \\
\text { streams by } \\
\text { putting } \\
\text { input } 1 \text { in } \\
\text { front of } \\
\text { input } 2\end{array}$ & \\
\hline Repack Bits & 1 byte & 8 bytes & $\begin{array}{l}\text { Length tag name: Input } \\
\text { the length of the data } \\
\text { stream, Packet } \\
\text { Alignment: How } \\
\text { packets are aligned, } \\
\text { Endianness MSB or } \\
\text { LSB (which one comes } \\
\text { first in stream) }\end{array}$ & $\begin{array}{l}\text { Converts } \\
\text { the stream } \\
\text { of bytes } \\
\text { into a } \\
\text { stream of } \\
\text { bits }\end{array}$ & $\begin{array}{l}\text { Modulation } \\
\text { techniques } \\
\text { deal with bits } \\
\text { and not bytes }\end{array}$ \\
\hline
\end{tabular}


Table 4. Functionalities of blocks during signal modulation steps

\begin{tabular}{|c|c|c|c|c|c|}
\hline Block & Input & Output & Parameters & Actions & Purpose \\
\hline $\begin{array}{l}\text { Char to } \\
\text { float }\end{array}$ & $\begin{array}{l}\text { Charact } \\
\text { er }\end{array}$ & Float & $\begin{array}{l}\text { Scale: Ratio of Char value to } \\
\text { Float value }\end{array}$ & $\begin{array}{l}\text { Converts a } \\
\text { character value } \\
\text { of }-128 \text { to } 127 \\
(0 \text { and } 1 \text { in this } \\
\text { case as we are } \\
\text { in BITS) to its } \\
\text { numeric value }\end{array}$ & $\begin{array}{l}\text { Numbers are } \\
\text { sent through } \\
\text { the channel, } \\
\text { not characters }\end{array}$ \\
\hline $\begin{array}{l}\text { Rational } \\
\text { Resampler }\end{array}$ & 1 float & 600 floats & $\begin{array}{l}\text { Interpolation: How many } \\
\text { samples to add to the stream }\end{array}$ & $\begin{array}{l}\text { Creates a } \\
\text { digital square } \\
\text { wave out of } \\
\text { single points }\end{array}$ & $\begin{array}{l}\text { We use a } \\
\text { square wave to } \\
\text { transmit data }\end{array}$ \\
\hline $\begin{array}{l}\text { Moving } \\
\text { Average }\end{array}$ & $\begin{array}{l}\text { Input is } \\
\text { a } \\
\text { stream } \\
\text { of } \\
\text { floats }\end{array}$ & $\begin{array}{l}\text { Output is } \\
\text { the } \\
\text { moving } \\
\text { sum of } \\
\text { the last } \mathrm{N} \\
\text { samples, } \\
\text { scaled by } \\
\text { the scale } \\
\text { factor }\end{array}$ & $\begin{array}{l}\text { Length of averaging window, } \\
\text { number to divide by, max } \\
\text { number of iterations in } \\
\text { averaging }\end{array}$ & $\begin{array}{l}\text { Sums the last } N \\
\text { points, to be } \\
\text { divided by the } \\
\text { scaling factor. } \\
\text { This is }\end{array}$ & \\
\hline $\begin{array}{l}\text { Float to } \\
\text { Complex }\end{array}$ & Float & Complex & Scale factor & $\begin{array}{l}\text { Converts float } \\
\text { to complex } \\
\text { number } \\
\text { (magnitude) }\end{array}$ & $\begin{array}{l}\text { Succeeding } \\
\text { blocks use } \\
\text { complex } \\
\text { numbers }\end{array}$ \\
\hline $\begin{array}{l}\text { Red } \\
\text { Pitaya } \\
\text { Sink }\end{array}$ & $\begin{array}{l}\text { Comple } \\
\mathrm{x}\end{array}$ & None & $\begin{array}{l}\text { Address: IP address of red } \\
\text { pitaya to connect to; Port: } \\
\text { Connection port; Center } \\
\text { frequency: Frequency by which } \\
\text { the red pitaya multiplies the } \\
\text { square wave by (important as } \\
\text { this is the frequency at which } \\
\text { the transducer oscillates); } \\
\text { Sampling rate: Sampling rate of } \\
\text { the red pitaya (basically we } \\
\text { want this as high as possible to } \\
\text { give the most accurate } \\
\text { waveform) }\end{array}$ & $\begin{array}{l}\text { Transmits the } \\
\text { data to the } \\
\text { transducer }\end{array}$ & $\begin{array}{l}\text { Acts as a } \\
\text { digital } \\
\text { (discrete) to } \\
\text { analog } \\
\text { (continuous) } \\
\text { converter and } \\
\text { a fir filter }\end{array}$ \\
\hline $\begin{array}{l}\text { Red } \\
\text { Pitaya } \\
\text { Source }\end{array}$ & None & Complex & $\begin{array}{l}\text { Address: IP address of red } \\
\text { pitaya to connect to; Port: } \\
\text { Connection port; Center } \\
\text { frequency: Frequency by which } \\
\text { the red pitaya multiplies the } \\
\text { square wave by (important as }\end{array}$ & $\begin{array}{l}\text { Receives the } \\
\text { data from the } \\
\text { transducer }\end{array}$ & $\begin{array}{l}\text { Acts as an } \\
\text { analog } \\
\text { (continuous) to } \\
\text { digital } \\
\text { (discrete) } \\
\text { converter and }\end{array}$ \\
\hline
\end{tabular}




\begin{tabular}{|c|c|c|c|c|c|}
\hline & & & $\begin{array}{l}\text { this is the frequency at which } \\
\text { the pzt oscillates); Sampling } \\
\text { rate: Sampling rate of the red } \\
\text { pitaya (basically we want this as } \\
\text { high as possible to give the } \\
\text { most accurate waveform) }\end{array}$ & & a fir filter \\
\hline $\begin{array}{l}\text { Low Pass } \\
\text { Filter }\end{array}$ & $\begin{array}{l}\text { Comple } \\
\mathrm{x}\end{array}$ & Complex & $\begin{array}{l}\text { Cutoff frequency: Frequency to } \\
\text { attenuate }\end{array}$ & $\begin{array}{l}\text { Attenuates } \\
\text { above a } \\
\text { threshold } \\
\text { frequency }\end{array}$ & $\begin{array}{l}\text { Removes high } \\
\text { frequency } \\
\text { noise as well } \\
\text { as any carrier } \\
\text { frequency to } \\
\text { leave square } \\
\text { wave }\end{array}$ \\
\hline $\begin{array}{l}\text { Active } \\
\text { Gain } \\
\text { Control } \\
\text { (AGC) }\end{array}$ & $\begin{array}{l}\text { Comple } \\
\mathrm{x}\end{array}$ & Complex & $\begin{array}{l}\text { The gain is updated by the } \\
\text { equation: Gain = Gain + Rate * } \\
\text { (Reference - abs(Input)); Max } \\
\text { Gain: the automatic gain will } \\
\text { not exceed this value. If set to } 0 \\
\text { there is no max }\end{array}$ & $\begin{array}{l}\text { Applies gain } \\
\text { loop onto signal }\end{array}$ & $\begin{array}{l}\text { Stabilizes the } \\
\text { signal for } \\
\text { demodulation }\end{array}$ \\
\hline $\begin{array}{l}\text { AM } \\
\text { Demod }\end{array}$ & $\begin{array}{l}\text { Comple } \\
\mathrm{x}\end{array}$ & Float & $\begin{array}{l}\text { Channel_rate - incoming } \\
\text { sample rate of the AM } \\
\text { baseband; audio_decim - input } \\
\text { to output decimation rate } \\
\text { (integer); audio_pass - audio } \\
\text { low pass filter passband } \\
\text { frequency (float); audio_stop - } \\
\text { audio low pass filter stop } \\
\text { frequency (float) }\end{array}$ & $\begin{array}{l}\text { Demodulates } \\
\text { the signal }\end{array}$ & $\begin{array}{l}\text { Decimates the } \\
\text { square wave }\end{array}$ \\
\hline $\begin{array}{l}\text { Multiply } \\
\text { Const }\end{array}$ & Float & Float & Number to multiply by & $\begin{array}{l}\text { Multiplies by a } \\
\text { constant }\end{array}$ & Fine tuning \\
\hline Add Const & Float & Float & Number to add & Adds a number & Fine tuning \\
\hline Threshold & $\begin{array}{l}\text { Float } \\
\text { (range } \\
\text { of } \\
\text { values) }\end{array}$ & $\begin{array}{l}\text { Float }(1 \\
\text { or } 0)\end{array}$ & $\begin{array}{l}\text { Upper threshold, lower } \\
\text { threshold }\end{array}$ & $\begin{array}{l}\text { The output } \\
\text { transitions from } \\
0.0 \text { to } 1.0 \text { when } \\
\text { the input signal } \\
\text { transitions from } \\
\text { below to above } \\
\text { the High level. } \\
\text { The output } \\
\text { transitions from } \\
1.0 \text { to } 0.0 \text { when } \\
\text { the input signal } \\
\text { transitions from } \\
\text { above to below } \\
\text { the Low level. }\end{array}$ & $\begin{array}{l}\text { The thereshold } \\
\text { converts the } \\
\text { stream of } \\
\text { floats into } \\
\text { binary (bits) }\end{array}$ \\
\hline
\end{tabular}


Table 5. Functionalities of blocks after signal modulation steps

\begin{tabular}{|c|c|c|c|c|c|}
\hline Block & Input & Output & Parameters & Actions & Purpose \\
\hline Map & Float & Float & $\begin{array}{l}\text { Map of values to send } \\
\text { to }\end{array}$ & $\begin{array}{l}\text { Maps } \\
\text { incoming data } \\
\text { to } 0 \text { or } 1\end{array}$ & $\begin{array}{l}\text { Makes sure } \\
\text { that data is } \\
\text { mapped to bits }\end{array}$ \\
\hline $\begin{array}{l}\text { Repack } \\
\text { Bits }\end{array}$ & 8 bytes & 1 byte & $\begin{array}{l}\text { Length tag name: } \\
\text { Input the length of the } \\
\text { data stream, Packet } \\
\text { Alignment: How } \\
\text { packets are aligned, } \\
\text { Endianness MSB or } \\
\text { LSB (which one } \\
\text { comes first in stream) }\end{array}$ & $\begin{array}{l}\text { Converts the } \\
\text { information } \\
\text { from bits into } \\
\text { bytes }\end{array}$ & $\begin{array}{l}\text { Byte } \\
\text { manipulation } \\
\text { technique }\end{array}$ \\
\hline $\begin{array}{l}\text { Packed to } \\
\text { Unpacked }\end{array}$ & 1 byte & 8 bytes & $\begin{array}{l}\text { Endianness MSB or } \\
\text { LSB (which one } \\
\text { comes first in stream) }\end{array}$ & $\begin{array}{l}\text { Converts the } \\
\text { bytes into bits }\end{array}$ & $\begin{array}{l}\text { The correlate } \\
\text { access code } \\
\text { deals with bits, } \\
\text { not bytes }\end{array}$ \\
\hline $\begin{array}{l}\text { Correlate } \\
\text { Access } \\
\text { Code }\end{array}$ & $\begin{array}{l}\text { Continuous } \\
\text { stream of bits }\end{array}$ & $\begin{array}{l}\text { Continuous } \\
\text { stream of bits } \\
\text { without } \\
\text { header bits }\end{array}$ & $\begin{array}{l}\text { Preamble and Access } \\
\text { Code: What the } \\
\text { correlate access code } \\
\text { will look for when } \\
\text { assessing what is a } \\
\text { packet }\end{array}$ & $\begin{array}{l}\text { Removes the } \\
\text { header bits } \\
\text { from the bit } \\
\text { stream }\end{array}$ & $\begin{array}{l}\text { The original } \\
\text { data has no } \\
\text { header bits, so } \\
\text { we want to } \\
\text { remove them }\end{array}$ \\
\hline $\begin{array}{l}\text { Repack } \\
\text { Bits }\end{array}$ & 8 bytes & 1 byte & $\begin{array}{l}\text { Length tag name: } \\
\text { Input the length of the } \\
\text { data stream, Packet } \\
\text { Alignment: How } \\
\text { packets are aligned, } \\
\text { Endianness MSB or } \\
\text { LSB (which one } \\
\text { comes first in stream) }\end{array}$ & $\begin{array}{l}\text { Converts the } \\
\text { bits into bytes }\end{array}$ & $\begin{array}{l}\text { Original data } \\
\text { was stored as } \\
\text { characters, not } \\
\text { bits }\end{array}$ \\
\hline File Sink & $\begin{array}{l}\text { Continuous } \\
\text { stream of } \\
\text { characters }\end{array}$ & None & $\begin{array}{l}\text { Input type: What kind } \\
\text { of information is } \\
\text { being written to file; } \\
\text { Unbuffered: Whether } \\
\text { or not the file is being } \\
\text { buffered; Append file: } \\
\text { Whether the incoming } \\
\text { data overwrites the } \\
\text { old file or append it. }\end{array}$ & & $\begin{array}{l}\text { Saves the } \\
\text { incoming file }\end{array}$ \\
\hline
\end{tabular}




\subsection{Communication Protocol Testing and Analysis}

To compare different data transmission techniques, a simple message was passed through the system and monitored in each GNURadio flowchart block. In this example, we send a file with characters "!s!" for demonstration purposes. We will follow the journey of a packet as it is transmitted through the system. Below is a representation of what occurs at each step in the process.

In the file source block, we see how file is being disassembled into three values: 33, 115, 33. The value of 33 corresponds to “!” and the value of 115 corresponds to "s”. Figure 4 below displays the raw data and each character labeled as a separate color.
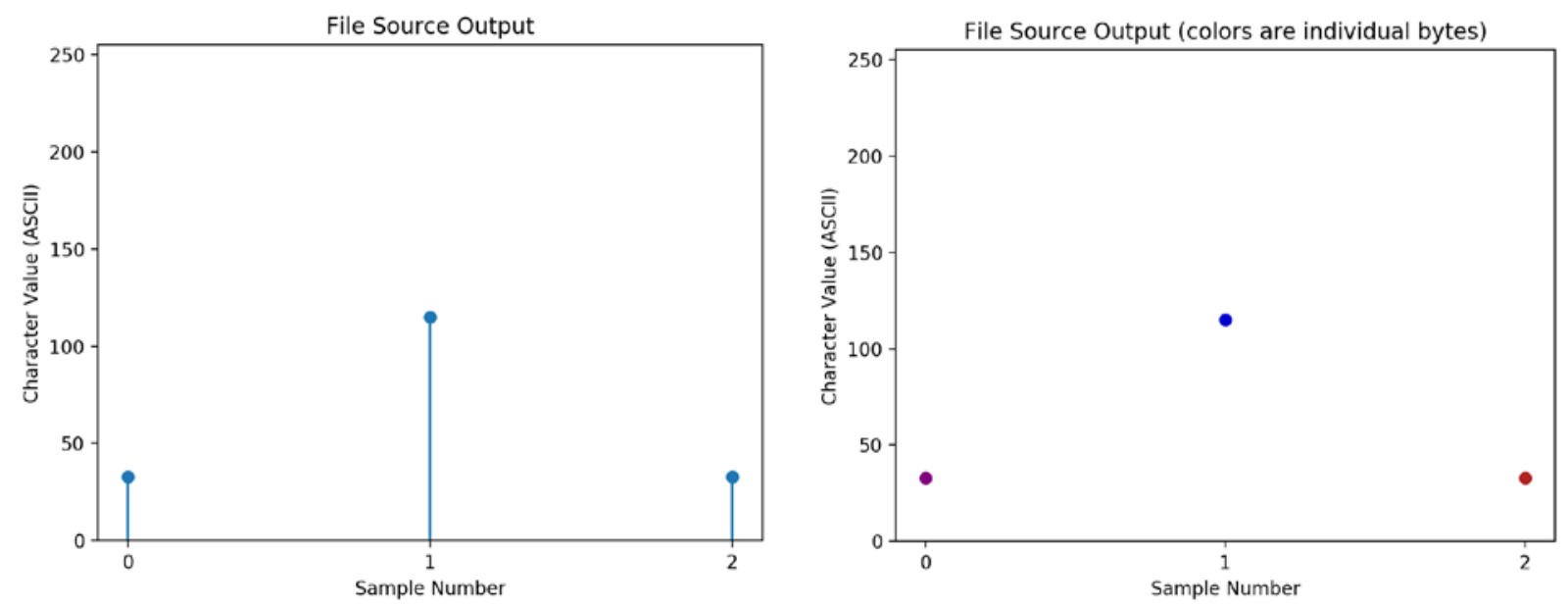

Figure 4 - File source output (left) raw data, (right) data colored as individual bytes

The protocol formatter block creates a tagged stream of 1's and 0's that will be attached to the payload. In our case, we use the default preamble of '1010010011110010' and access code of '1010110011011101101001001110001011110010100011000010000011111100', which is displayed above as bytes in Figure 5 (raw data and data colored as bytes) 

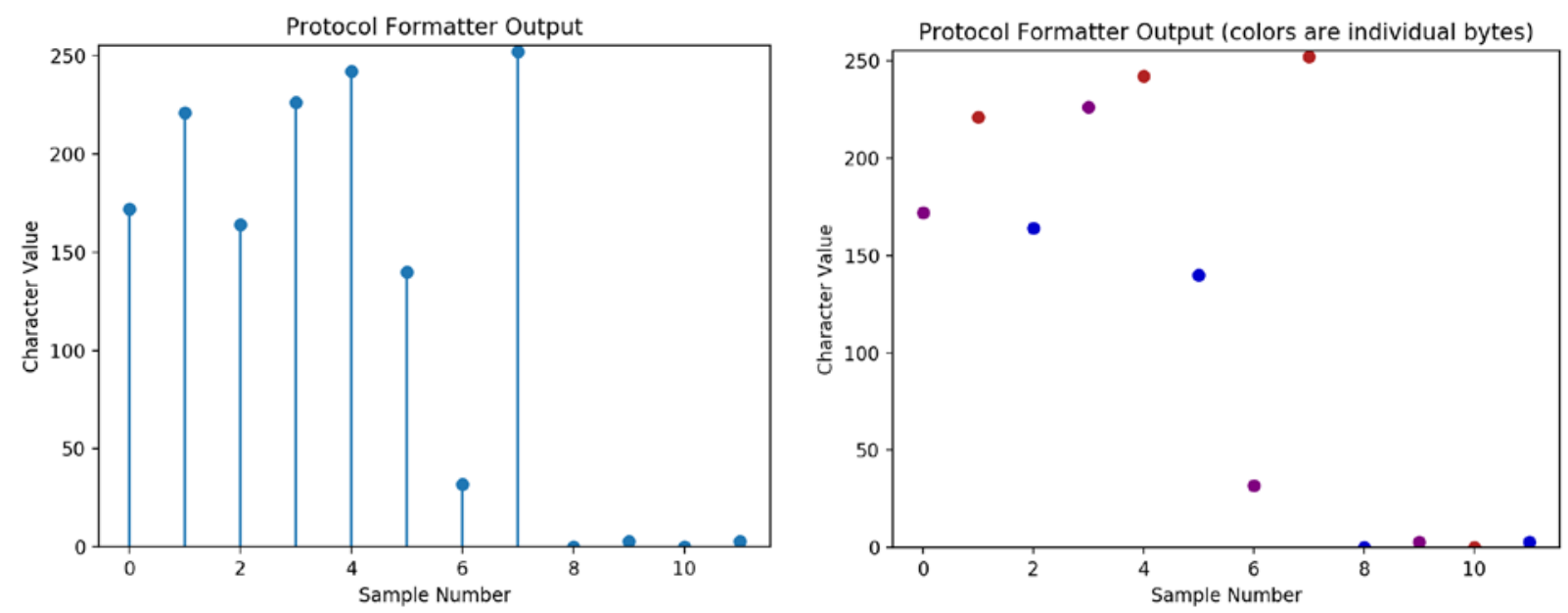

Figure 5 - Protocol formatter output (left) raw data, (right) data colored as individual bytes

Figure 6 below visualizes how the header file is combined with the payload. We first see the attached preamble in the form of bytes. The first 12 bytes are from the protocol formatter while the last 3 are from the file source. Specifically, we can see that bytes 12, 13, 14 match the file source.
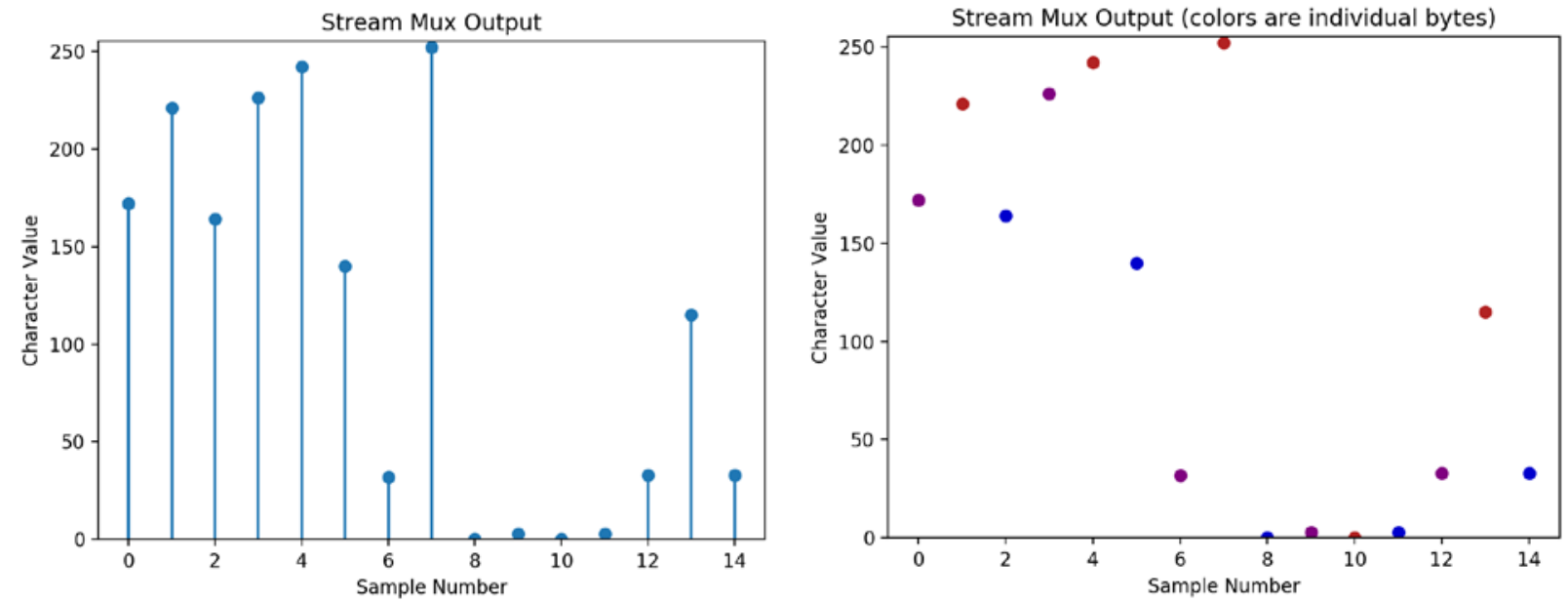

Figure 6 - Stream mux output (left) raw data, (right) data colored as individual bytes

We can now see how after the stream mux, we unpack the individual bytes into 8 bits each. The individual bytes are displayed above with colors corresponding to each byte in Figure 7 . There are 15 bytes in total for the packet, meaning that we are now sending 120 samples through the system $(15 * 8=120)$ 

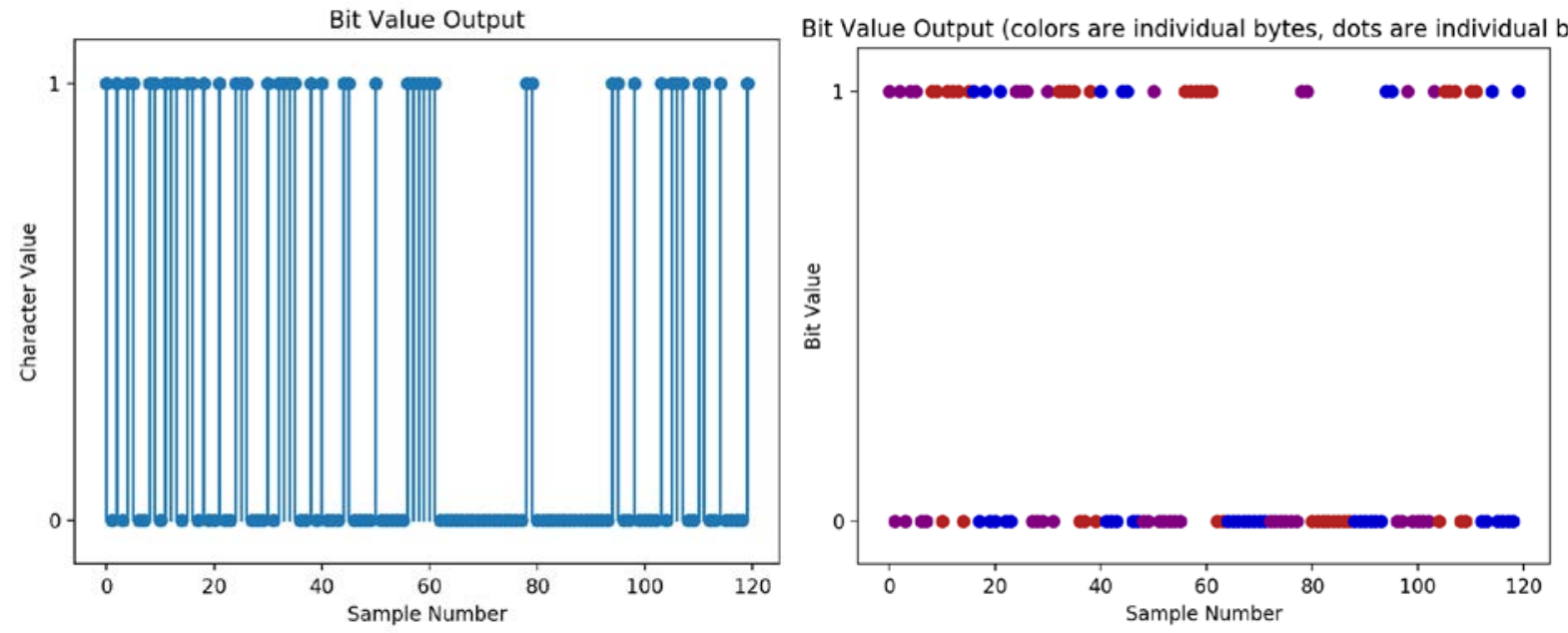

Figure 7 - Bit value output (left) raw data, (right) data colored as individual bytes

Figure 8 below shows a zoomed-in view of the first byte in the stream. We can see that it does in fact correspond to the character value of 172 , since $10101100=\left(1 \times 2^{7}\right)+\left(0 \times 2^{6}\right)+\left(1 \times 2^{5}\right)+(0$ $\left.\times 2^{4}\right)+\left(1 \times 2^{3}\right)+\left(1 \times 2^{2}\right)+\left(0 \times 2^{1}\right)+\left(0 \times 2^{0}\right)=172$.
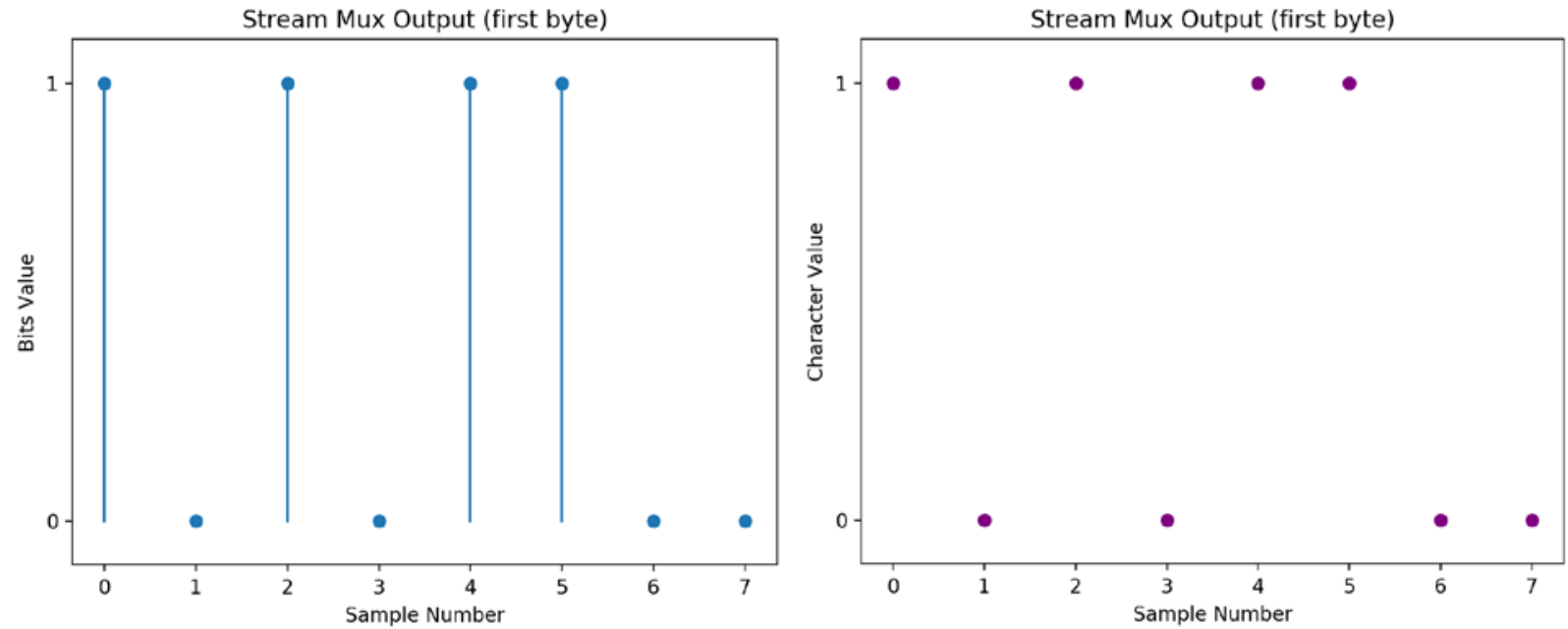

Figure 8 - First byte stream mux output (left) raw data, (right) data colored as individual bytes

Figure 9 below shows what happens to the information after the rational resampler and moving average block. Each bit is interpolated to create 600 bits after it, which is why the total number of samples goes from 120 in the figure with the data displayed as bits, to 72000 in the above figures $(120 * 600=72000)$. In both graphs, we can see how the signal is converted from digital to analog, transforming the latter into a square wave. 

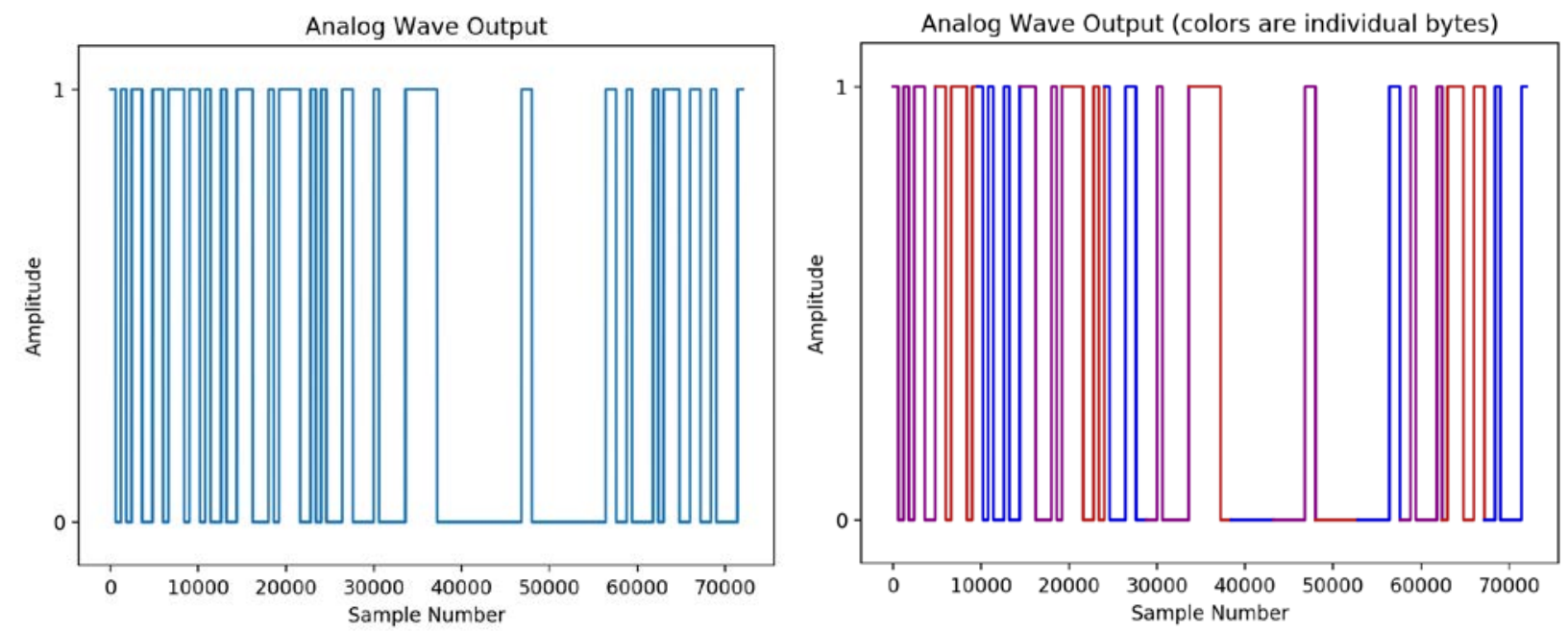

Figure 9 - Analog wave output (left) raw data, (right) data colored as individual bytes

Figure 10 below displays Fast Fourier Transform (FFT) digital calculation of the analog wave output spectrum. The bandwidth of the signal is less than $50 \mathrm{KHz}$.

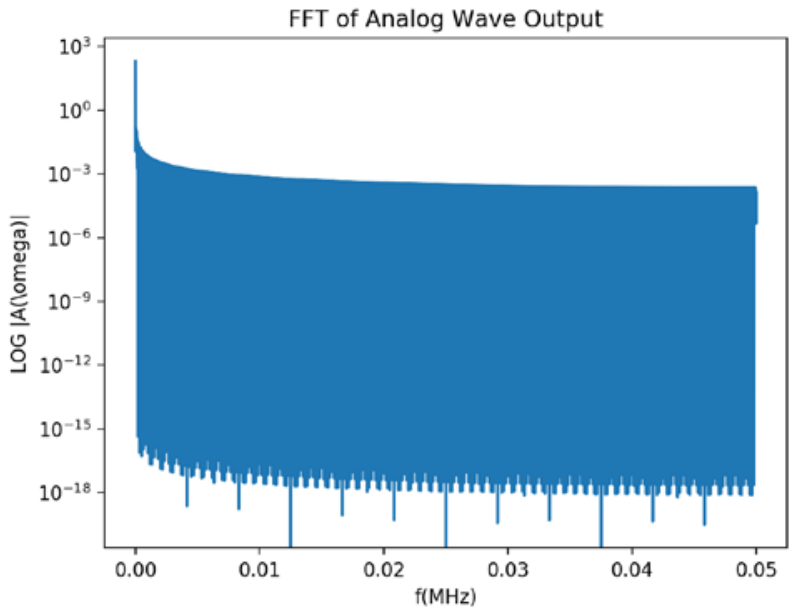

Figure 10 - FFT spectrum of analog wave output

Figure 11 below displays the data taken from the oscilloscope at a sampling rate of $25 \mathrm{Ms} / \mathrm{s}$, which is much higher than GNURadio's rate of $100 \mathrm{ks} / \mathrm{s}$, which is why the number of samples for the same signals is 250 times higher than in GNURadio $(250 * 100,000=25,000,000)$. The individual bytes from the square wave are all translated into a signal with amplitude ranging from -0.3 to 0.3 volts. Note that in our setup we pass this signal into a $50 \mathrm{~dB}$ amplifier, the transducer actually feels a voltage of about 95 volts. We can see that Red Pitaya introduces ringing into the system on every on/off voltage high/low. This kind of behavior is why we chose to use binary ASK over quad ASK, since this kind of instability only lends itself to on or off keying. 

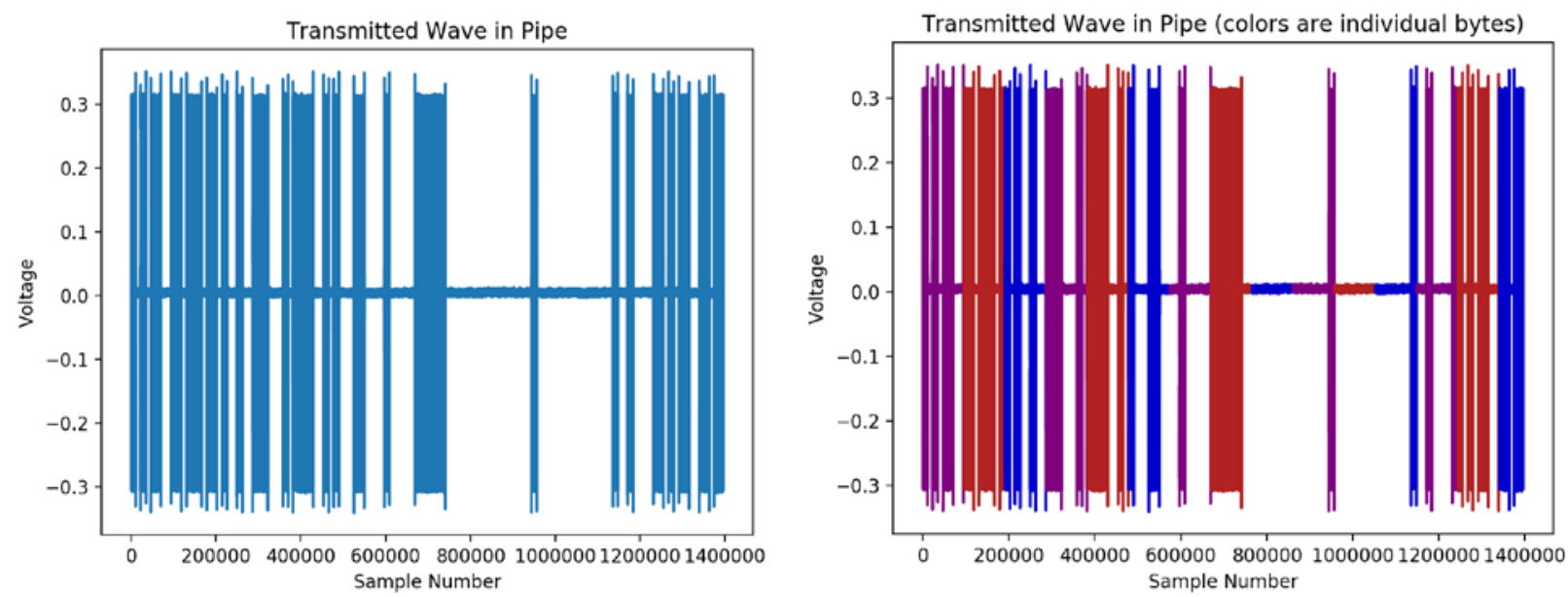

Figure 11 - Transmitted wave on pipe (left) raw data, (right) data colored as individual bytes

Figure 12 below displays FFT spectrum of the transmitted signal on pipe. Compared to the spectrum of the analog output signal in Figure 10, we see that the frequency response of the square wave is still seen on the lower end of the spectrum, and we can also clearly see the carrier frequency centered at $1.8 \mathrm{MHz}$. We can see that the frequency response of the carrier frequency is stronger than the frequency response of the base band.

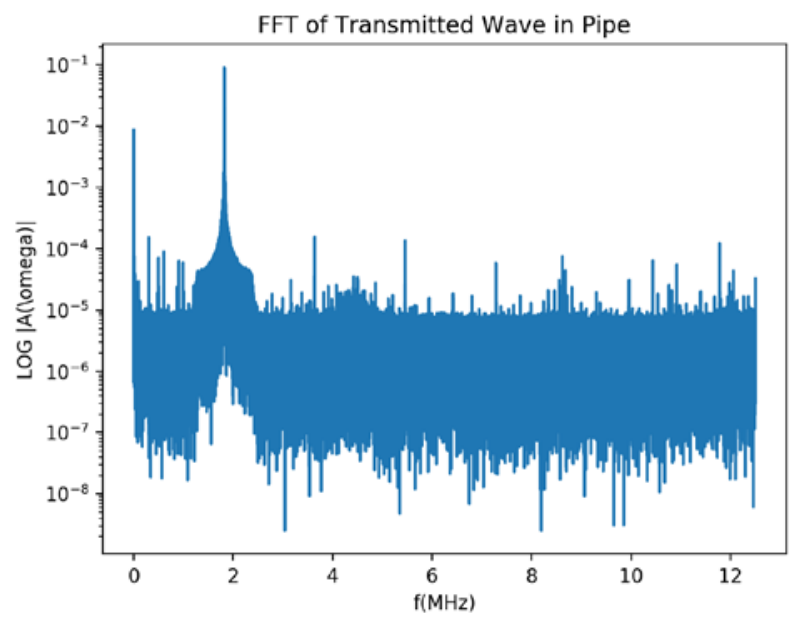

Figure 12 - FFT spectrum of transmitted wave of pipe

Figure 13 displays the received signal waveform before demodulation. The data is obtained from the oscilloscope, which is now connected to the output of the low noise amplifier placed after the receiving transducer. We can see that the signal, even with the use of two amplifiers, is attenuated by 10 and in addition, a DC offset of -0.075 volts is added to the signal. Compared to the 
transmitted signal, we can see more distortion along the peaks of the signal, as well as a much larger amount of noise around the DC offset.
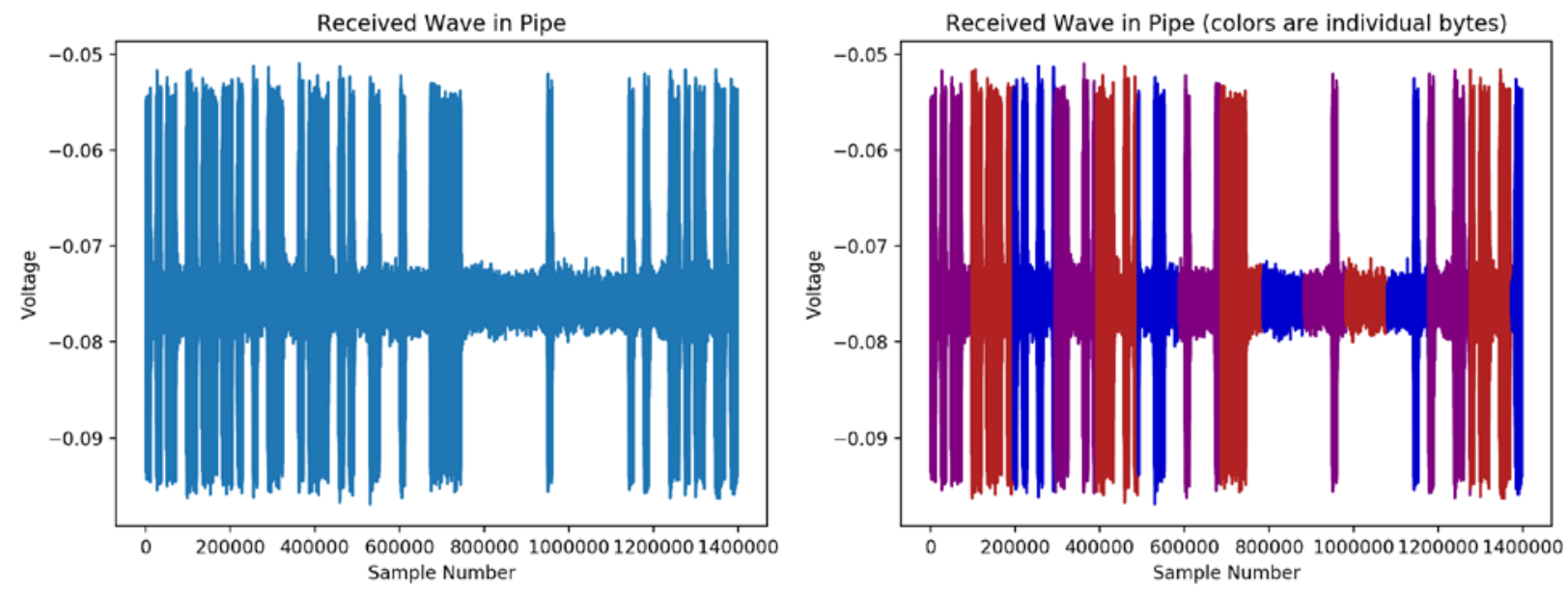

Figure 13 - Received wave on pipe before demodulation (left) raw data, (right) data colored as individual bytes

Figure 14 displays the frequency spectrum of the received waveform before demodulation. Compared to the transmitted signal spectrum shown in Figure 12, we can see that the frequency response of the baseband is higher than the frequency response of the carrier frequency.

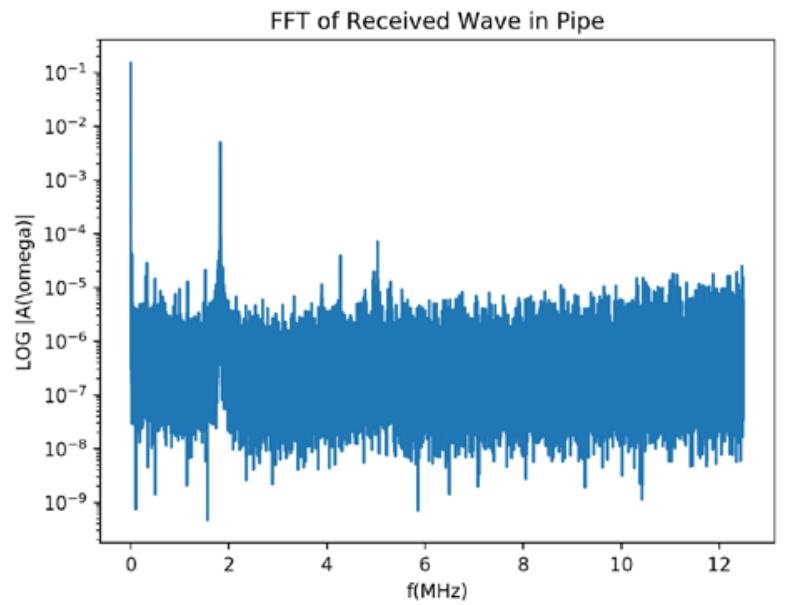

Figure 14 - FFT spectrum of received wave on pipe before demodulation

Figure 15 below displays of the first byte in the string of characters in the received signal in Figure 13. We see that after the shift keying has been switched to the off position, we still see some ringing from the waveform. 


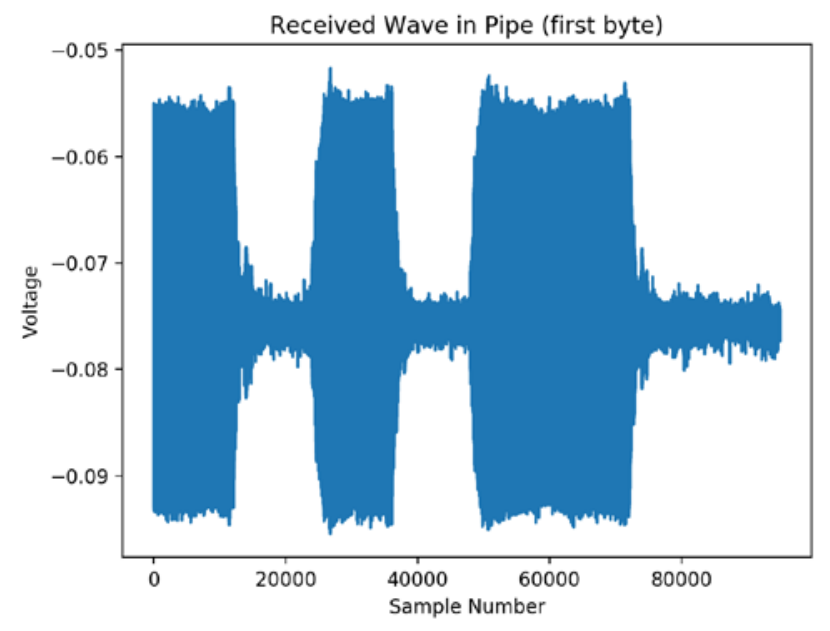

Figure 15 - First byte in received signal before demodulation

Figure 16 below is the received demodulated signal with Red Pitaya board, displaying the repeated string of characters. We see that at first the system produces information that cannot be decoded (Sample number $<500000$ ), but then proceeds to produce a waveform that is more stable. Since our sample rate is $100000 \mathrm{~S} / \mathrm{s}$, we can therefore conclude that the system has to transmit for 5 seconds before the information can be processed successfully. The overall shape of the incoming signal envelope has a sinusoidal shape, suggesting that there is a phase mismatch between the transmitting Red Pitaya and the receiving Red Pitaya boards.

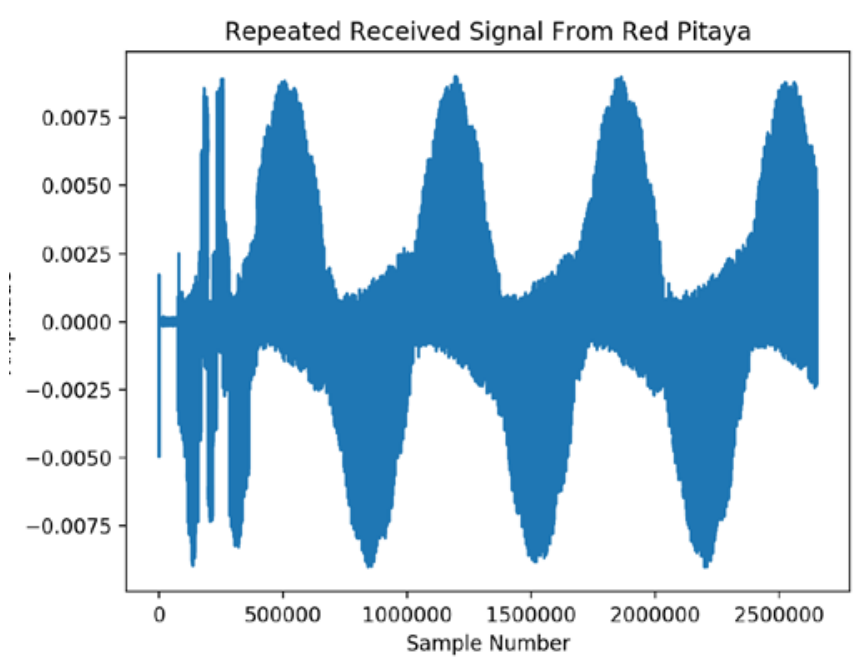

Figure 16 - Received demodulated signal with Red Pitaya board

Figure 17 below displays FFT spectrum of the demodulated received signal, which should be matching that of the transmitted analog square wave in Figure 10. We can see that the frequency 
roll off is similar to that of the transmitted information, however the frequency response is not as smooth as in the transmitted signal.

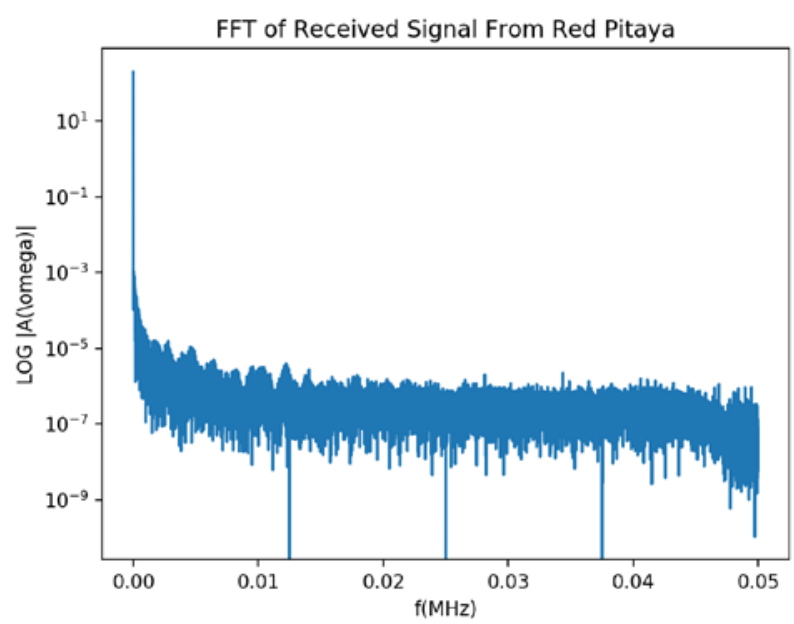

Figure 17 - FFT spectrum of demodulated signal received with Red Pitaya board

Figure 18 below provides a zoomed in version of the character transmission of the received signal from Red Pitaya board. If we compare this plot to the transmitted waveform in Figure 11, we can see that there is much more ringing in the received signal compared to the transmitted one. This result matches that of the frequency response, as we can see that the imbalanced frequency response makes the square waves less defined.
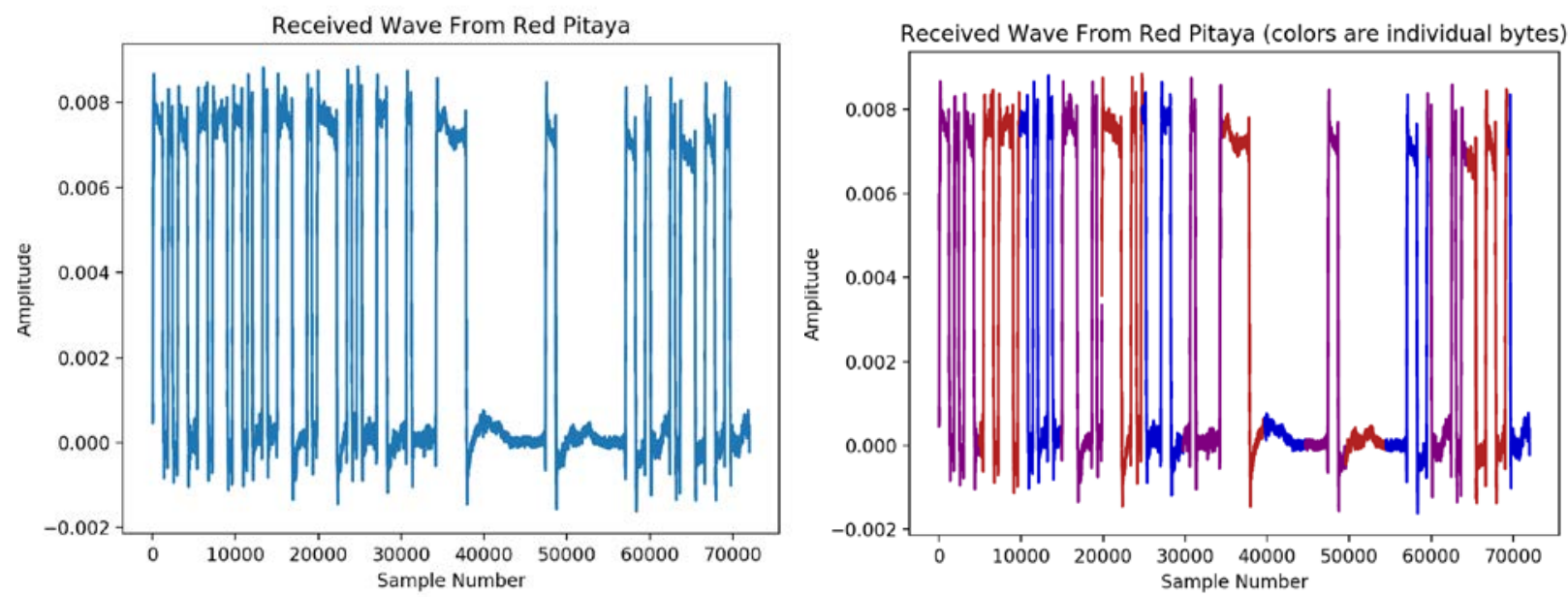

Figure 18 - Zoomed-in received signal from Red Pitaya board (left) raw data, (right) data colored as individual bytes

Figure 19 below displays a plot of the signal as it passes through the automatic gain control (AGC). We can see that this signal has the same shape as that of the signal that exits Red Pitaya, however 
the amplitude is increased. This is done to prepare the signal for the threshold section, which needs a greater difference in amplitude between on and off states representing digital " 0 " and " 1 "

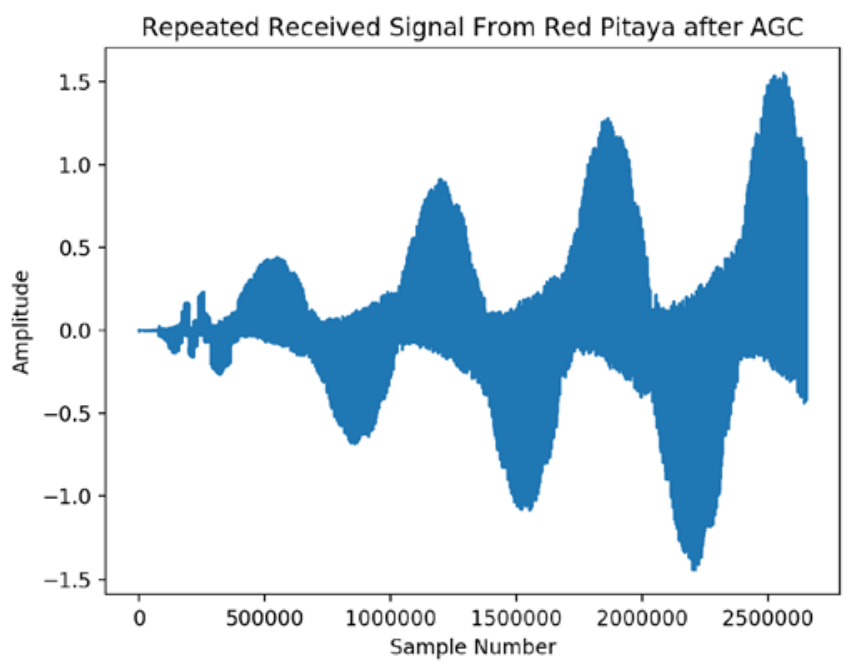

Figure 19 - Signal amplification with automatic gain control (AGC)

AGC is needed to compensation of demodulated signal amplitude reduction due to random phase difference in the received and reference waveforms. The average or peak output signal level is used to dynamically adjust the gain of the amplifiers, enabling the circuit to work satisfactorily with a greater range of input signal levels, so that we can set a proper up threshold for ' 1 ' and down threshold for ' 0 '. the AGC effectively reduces the amplitude if the signal is strong and raises it when it is weaker. Implements an automatic gain control with a single rate parameter for both attack and decay. The gain is updated by the equation

$$
\text { Gain }=\text { Gain }+ \text { Rate } *(\text { Reference }- \text { abs }(\text { Input }))
$$

The equation is for each sample.

- Attack rate - how fast the AGC decreases the gain when a loud signal appears

- Decay rate - how fast the AGC increases the gain when the loud signal is gone

- Reference - This is the level the AGC will try to maintain

Figure 20 below displays the data of threshold block after demodulation. We can see that the system can only begin to recognize the bits after a certain number of samples have passed, showing that the active gain control needs time to grow before being demodulated. 


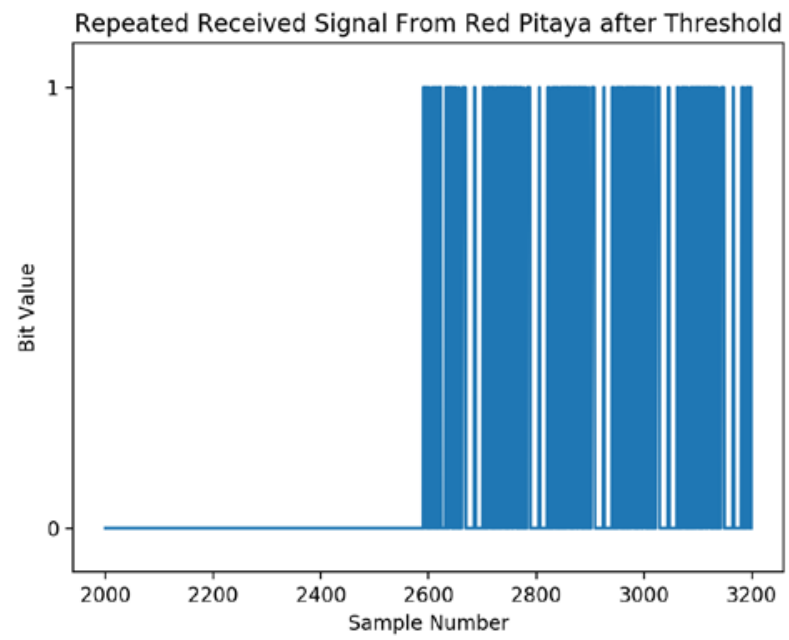

Figure 20 - Data of threshold block after demodulation

Figure 21 below displays information recovered from the threshold block after decimation. We see that the recovered information follows the same pattern as the transmitted bits.
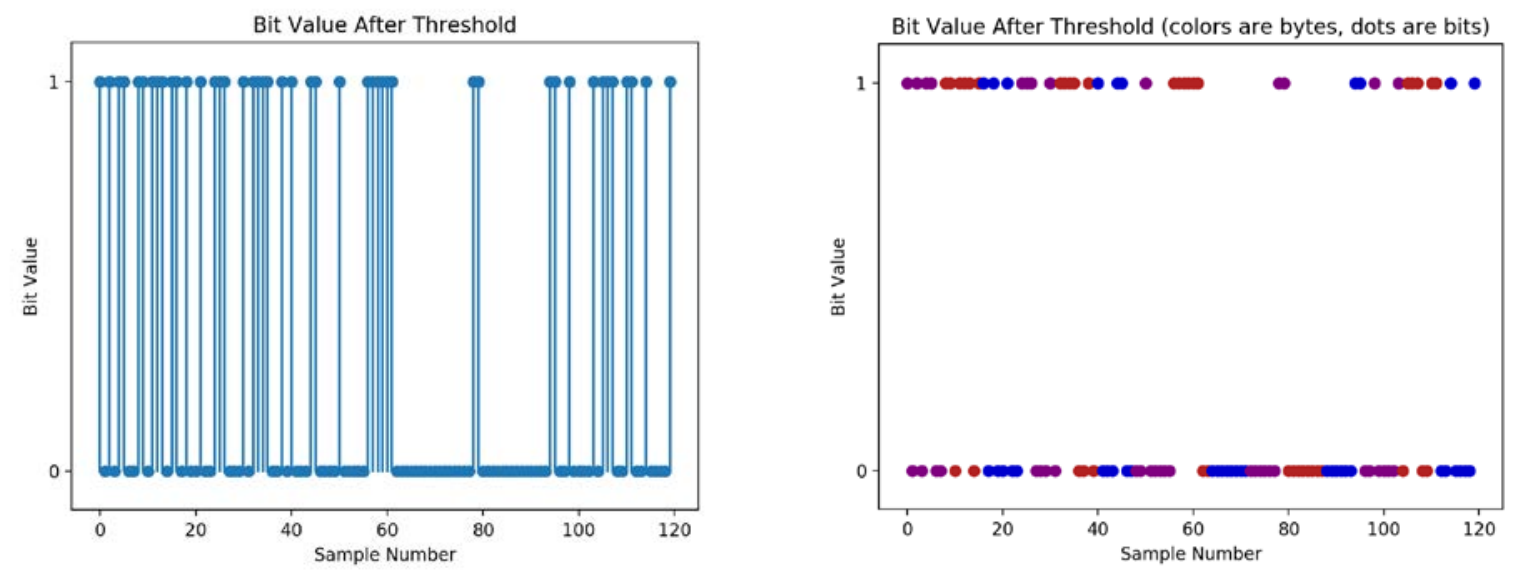

Figure 21 - Data recovered after decimation (left) raw data, (right) data colored as individual bytes

The correlate access code removes the bits from the stream that follow originate from the protocol formatter, leaving the original information. Figure 22 below displays the resulting data bits 

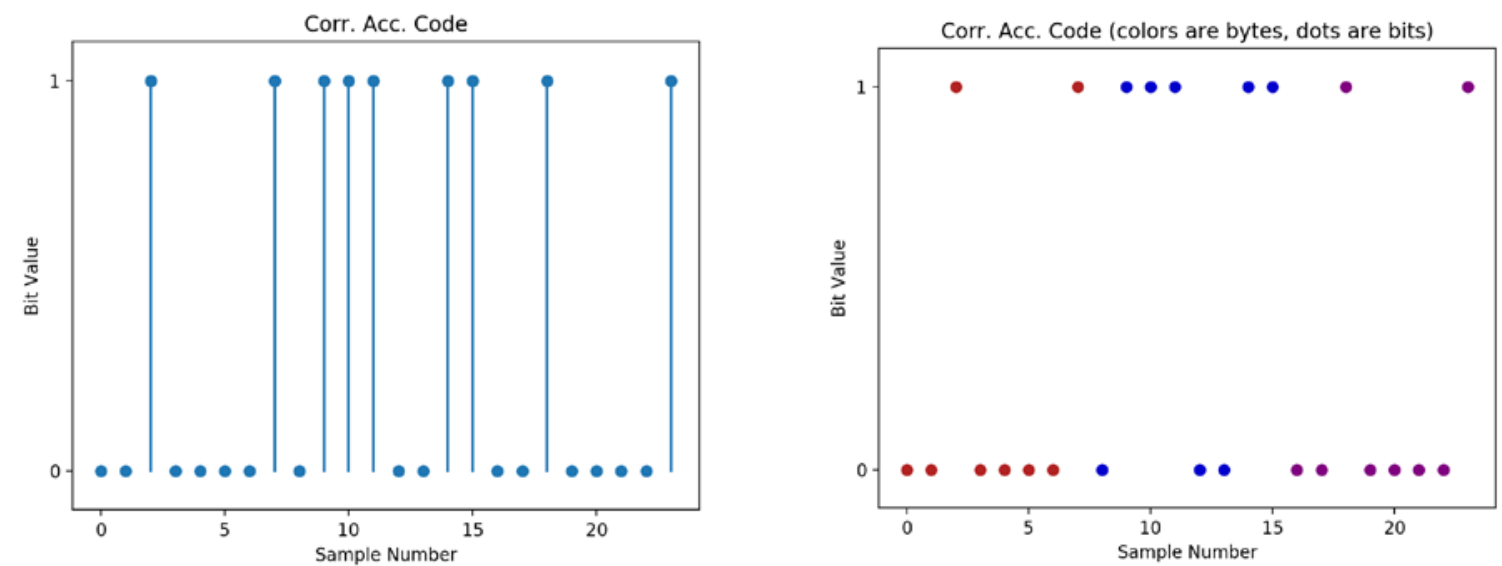

Figure 22 - Data recovered with correlated access code (left) raw data, (right) data colored as individual bytes

The repack bits block repacks the bits into bytes and stores the information into the file sink, successfully recovering the transmitted file. The data is displayed in Figure 23 below.
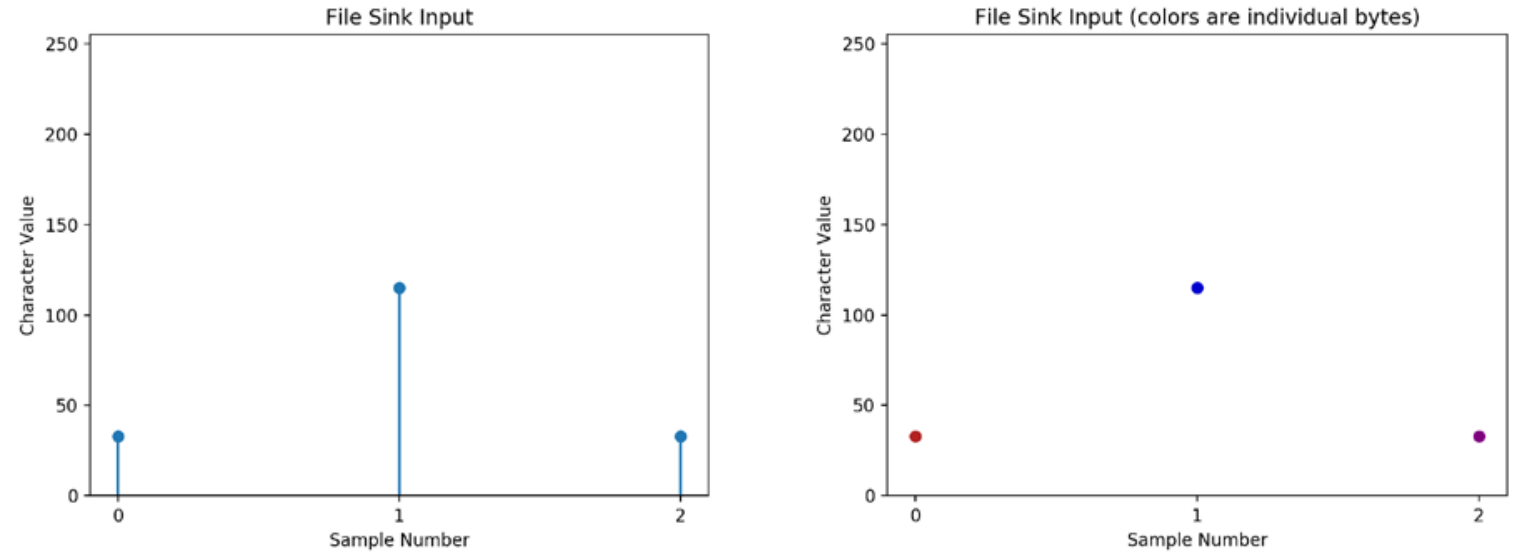

Figure 23 - Data from file sink input (left) raw data, (right) data colored as individual bytes

\subsection{Comparison of Communication Protocols Performance}

To quantify differences in performance of ASK and PSK communication protocols, we developed a Python script that transmitted a string of characters, similar to that of an image header file. Data was transmitted with different combinations of modulation and error correction. We then compared the number output by the file source against the input of the file sink to see how effectively the data was transmitted. 
The Python code considers 4 combinations of communication channels: ASK (Amplitude Shift Keying), ASK with CC (with Convolutional front error Correction), PSK (Phase Shift Keying), PSK with CC. We ran a simulation channel that modified the SNR (Signal to Noise Ratio) by adding various voltages of AWGN (Additive White Gaussian Noise). Figure 24 displays bit error rate (BER) in header file transmission vs. signal to noise ratio (SNR) in units of dB.

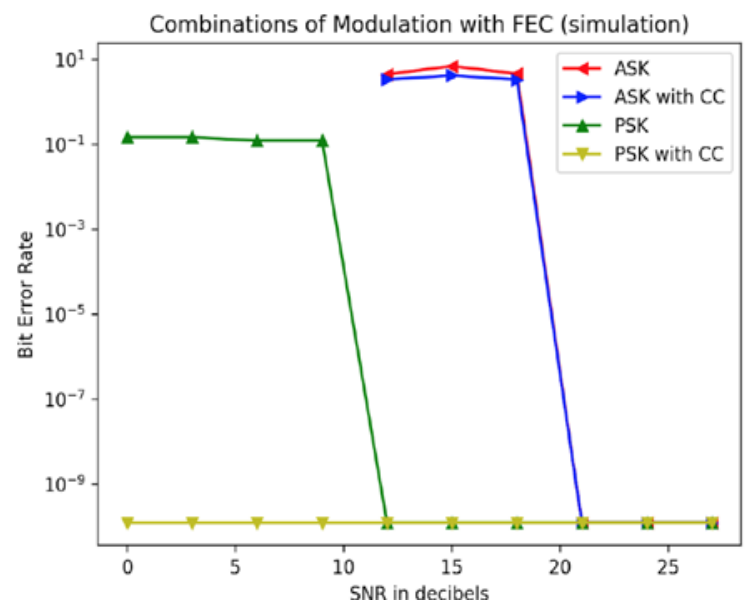

Figure 24 - BER vs. SNR for ASK, ASK with CC, PSK, and PSK with CC schemes

Overall, we see that the most effective method of communication in simulation is PSK with CC, which was successful at transmitting at all SNR levels. PSK was also effective, with the bit error rate dropping sharply after 10 decibels. The CC technique appears to slightly increase the performance of ASK modulation.

However, due to experimental limitations, the analog to digital converter was not capable of effectively demodulating the signal transmitted through the pipe. Therefore, instead ASK and ASK with CC were tested. The results are plotted in Figure 25 as BER vs carrier frequency. Interestingly, ASK either transmits perfectly or not at all at different frequencies while ASK with CC transmits at all frequencies, abet with a high error rate. Both techniques work at $2 \mathrm{MHz}$, which suggests that the communication system prefers higher frequencies. 


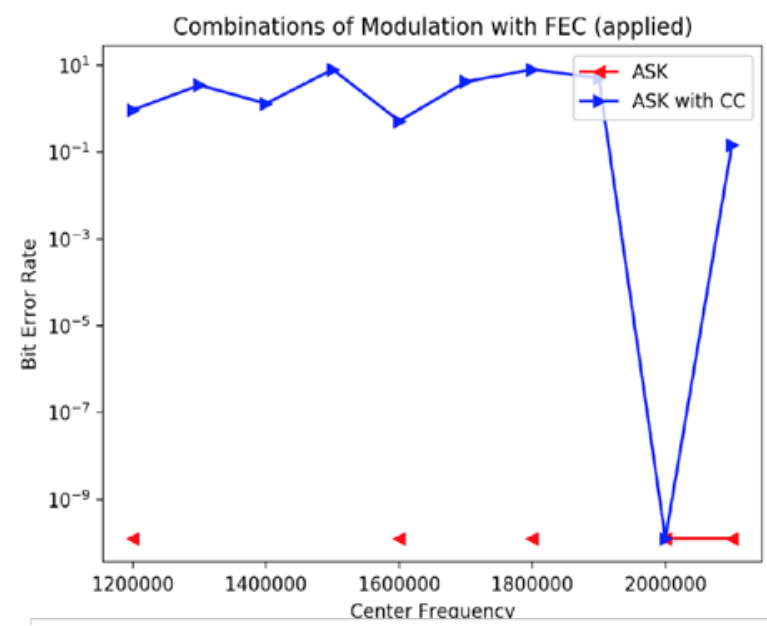

Figure 25 - BER vs. carrier frequency for different ASK and ASK with CC schemes

\subsection{Conclusion}

Overall, we can see the effect of each step in the GNURadio process for signal transmission. We see that passing the square wave through the communication channel leads to higher noise around the DC offset as well as a more jagged frequency response from the square wave. In order to further improve the system, we would have to lock the phase after the information is received from the Red Pitaya, as well as develop a system that helps reduce the number of samples the system needs to pass through the active gain loop before becoming decodable. 


\section{Demonstration of Information Transmission with Acoustic Communication System}

Using the communication protocol described in Section 2, we have demonstrated data transmission over a communication channel consisting of two PZT's on six-foot long stainless steel pipe. As described in previous reports, the parameters of the pipe in this study closely resembles those of the chemical volume control system (CVCS) charging line pipe, which penetrates through the containment building wall in some common reactor designs.

\subsection{Acoustic Transducer Frequency Selection}

As discussed in Section 1, higher frequency of the carrier acoustic wave allows for transmitting information at high bit rate. As illustrated below, information is encoded with D/A into a square wave waveform with duty cycle. As an example, time-domain waveform with duty cycle of $100 \mu \mathrm{s}$ and its spectrum calculated with FFT are shown in Figure 26.
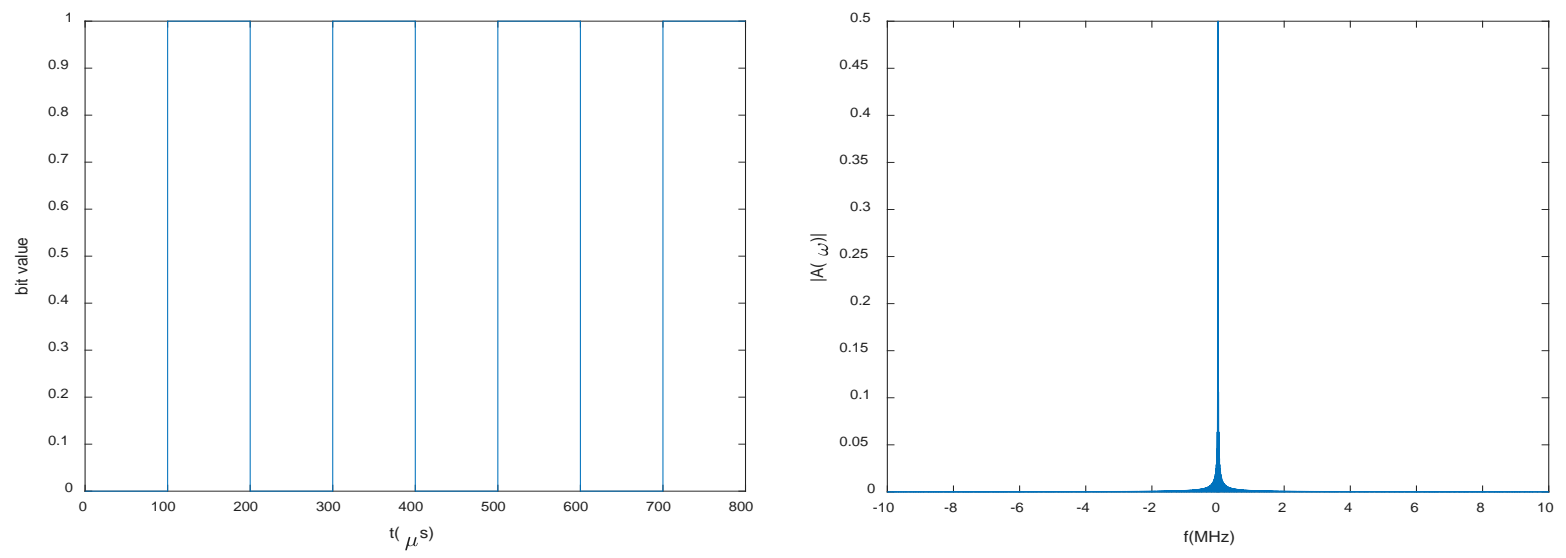

Figure 26 - Information-carrying waveform (left) time domain (right) frequency domain

The waveform is encoded as amplitude modulation of the carrier with acoustic frequency. Figure 27 shows amplitude modulated 2MHz carrier frequency and its FFT spectrum.
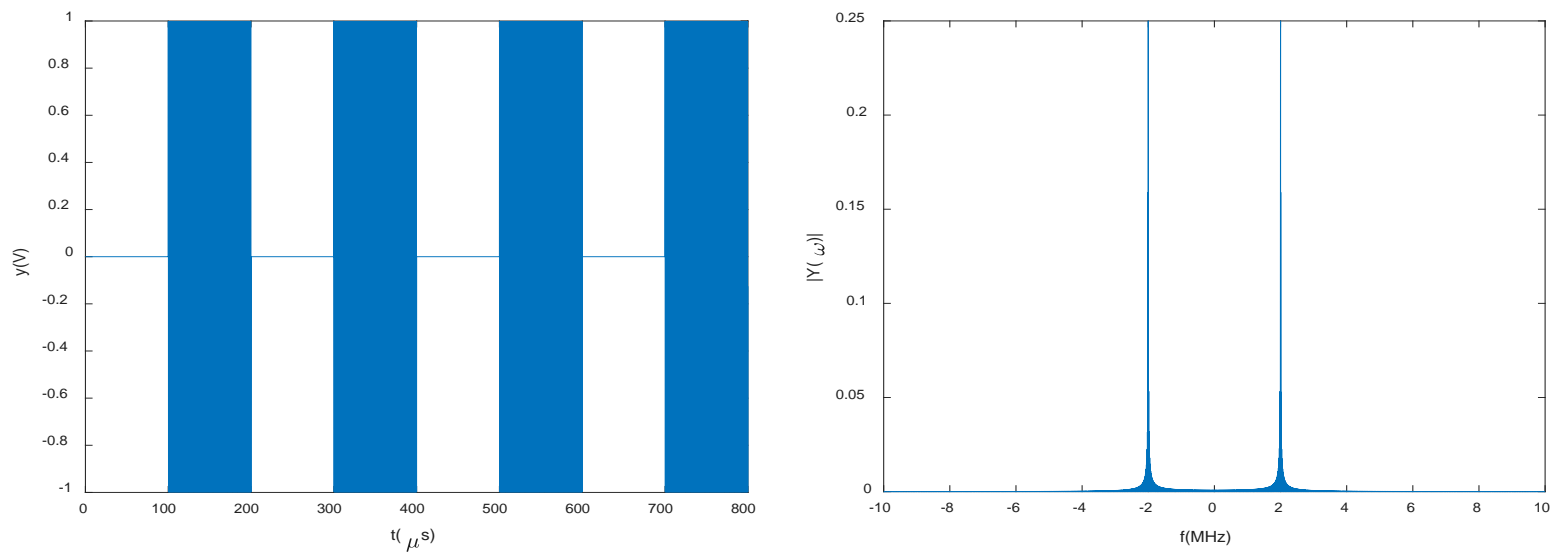

Figure 27 - Amplitude-modulated carrier waveform (left) time domain (right) frequency domain 
Demodulation at the receiver occurs by multiplying received signal by a reference sinusoid with the same acoustic frequency. The signal after multiplication and its FFT spectrum are shown in Figure 28.
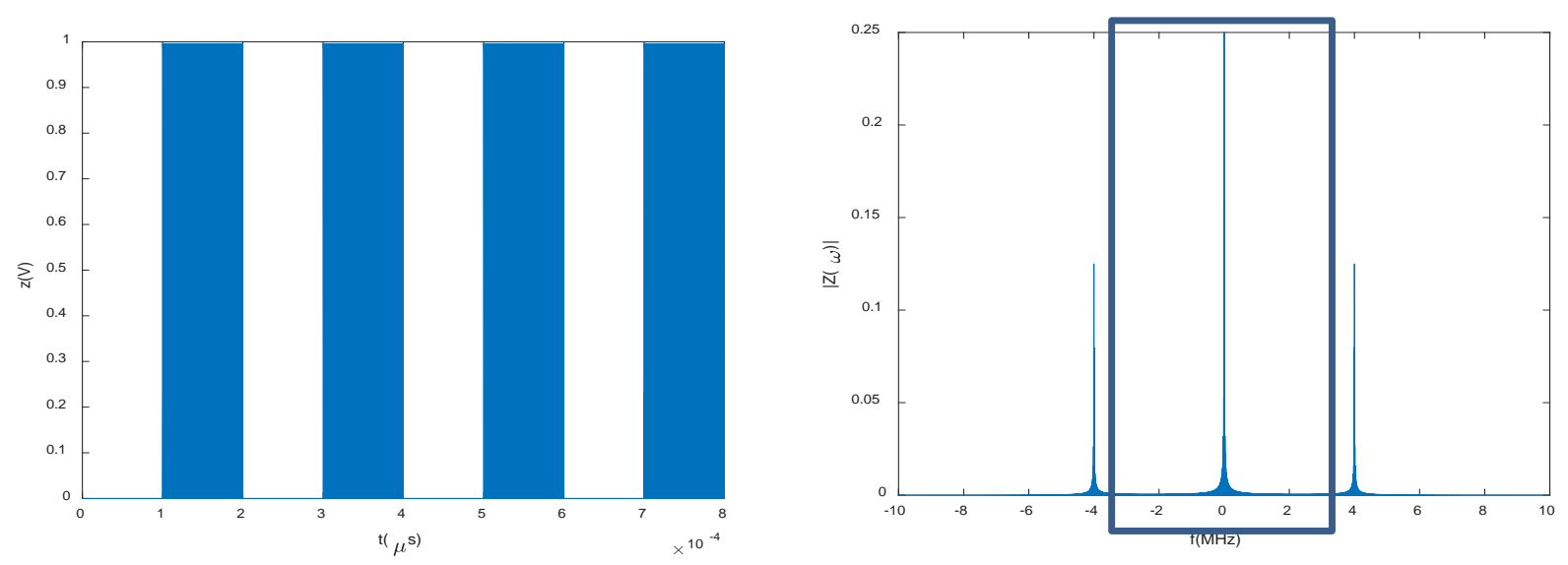

Figure 28 - Received signal multiplied by reference (left) time domain (right) frequency domain

Demodulation takes place by applying low-pass filter (LPF), shown as the box in the spectral plot, to remove carrier sidebands at $2 \mathrm{f}=4 \mathrm{MHz}$. The spectrum of information-carrying square wave is close to DC. For higher-frequency amplitude modulation (AM) square wave, the baseband spectrum will increase. The spectral width of the LPF or the frequency of the carrier will determine the limit of AM duty cycle. Thus information bit rate is proportional to frequency of the carrier $\mathrm{f}$.

At the same time, higher frequency results in larger absorption losses in the channel. A decrease in the plane harmonic wave amplitude as a result of its interaction with a medium takes place according to the Beer-Lambert law $e^{-\delta x}$, where $\mathrm{x}$ is the distance that the wave passes in the medium and $\delta$ is the damping coefficient. In what follows, the term "damping" will refer only to the wave amplitude decrease that is considered by an exponential multiplier, contrary to a decrease in the amplitude related to the wave front extension.

The damping factor consists of the coefficients of absorption $\delta_{1}$ and dissipation $\delta_{2}: \delta=\delta_{1}+\delta_{2}$. During absorption, a sound energy transforms to thermal, and during dissipation the energy remains sonic, but is emitted by a wave whose propagation is directional. Absorption is conditioned by ductility, elastic hysteresis (i.e., by a different elastic dependence during extension and compression), and heat conductivity.

The value of the dispersion coefficient in a medium is greatly affected by the correlation of the average size of heterogeneities and the average distance between heterogeneities with the elastic wavelength. In metals, the medium parameter that significantly affects the dispersion of crystals is the average size of a particle $\tilde{d}$. Then, total damping of stainless steel is calculated by the equation 


$$
\delta=A f+B f^{4} \tilde{d}
$$

Where A and B is the constant. The average size $\tilde{d}$ of stainless steel is $1.5 \mu \mathrm{m}$. Thus, the quadratic frequency term can be ignored, and attenuation in the channel is linearly proportional carrier frequency.

Commercially available PZT's are built for operation at a set of discrete frequency. In this study, considering tradeoffs between bit rate and attenuation, we have chosen PZT operating at $2 \mathrm{MHz}$. Losses incurred due to attenuation in the channel were compensated with power amplifier (transmitter side) and low-noise amplifiers (receiver side).

\subsection{Wedge Selection for PZT Refracted Shear Wave}

As described in Section 1, physical realization of communication channel with PZT requires generation of single mode wave. This can be accomplished via generating a refracted shear wave with PZT mounted on angled wedge. Commercially available plastic wedges have $30^{\circ}, 45^{\circ}$ and $60^{\circ}$ angles of incline. The first critical angle for plastic to stainless steel interface is approximately $29.5^{\circ}$, so that, in principle both $30^{\circ}, 45^{\circ}$ wedges are suitable. However, we have observed experimentally that using contoured $30^{\circ}$ wedge results in generating in both longitudinal and shear waves in the pipe. The modes were inferred from calculated velocities of pulses $(5790 \mathrm{~m} / \mathrm{s}$ and $3100 \mathrm{~m} / \mathrm{s}$ for longitudinal and shear wave velocities in stainless steel, respectively). Generation of longitudinal modes was possibly an artifact of relatively small radius of curvature of the pipe, such that parts of the wavefront were actually incident at the interface at angles smaller than the critical angle.

When using a $45^{\circ}$ wedge without contouring, we have observed that only shear waves were generated in the pipe. At the same time, the amplitude of the refracted shear wave was slightly smaller than that of generated with a contoured wedge. This is consistent with prior findings indicating that larger surface area of contact between wedge and pipe are results in better coupling of elastic wave energy into the pipe at the expense of reducing signal clarity.

For verification that only shear wave is generated, signals were measured on a stainless steel plate and stainless steel pipe of similar thicknesses. The same set of wedges and transducers separated by the same distances were used for plate and pipe measurements. Figure 29 displays the schematics of the experimental arrangement. A pair of $2 \mathrm{MHz}$ PZT transducers separated by a distance of $165 \mathrm{~cm}$ was used in this study. Transmitted pulses were of $200 \mu$ s time duration.

Comparison of time-domain signals transmitted on a plate and on a pipe are shown in Figure 30. Signal transmitted on a plate is shown in Figure 30(a), while those for transmission on a pipe are shown in Figures 30(b), (c) and (d). To validate COMSOL computer simulations predicting excitation of torsional waves on a pipe, receiver and transmitter at the ends of the pipe were aligned (Figure 30b), rotated by relative $90^{\circ}$ angle (Figure 30c), and rotated by relative $180^{\circ}$ angle (Figure $30 \mathrm{~d}$ ). The signal velocity obtained in all cases on plate and pipe was close to $3100 \mathrm{~m} / \mathrm{s}$ value, confirming that only shear wave modes were present in the channel. In addition, it appears that transmission is nearly insensitive to the relative angle between transmitter and receiver 
transducers, which confirms prediction of torsional wave excitation on a pipe. Insensitivity to alignment between transmitter and receiver is advantageous for communication system robustness, since exact alignment would be difficult to perform in field conditions at a nuclear power plant.

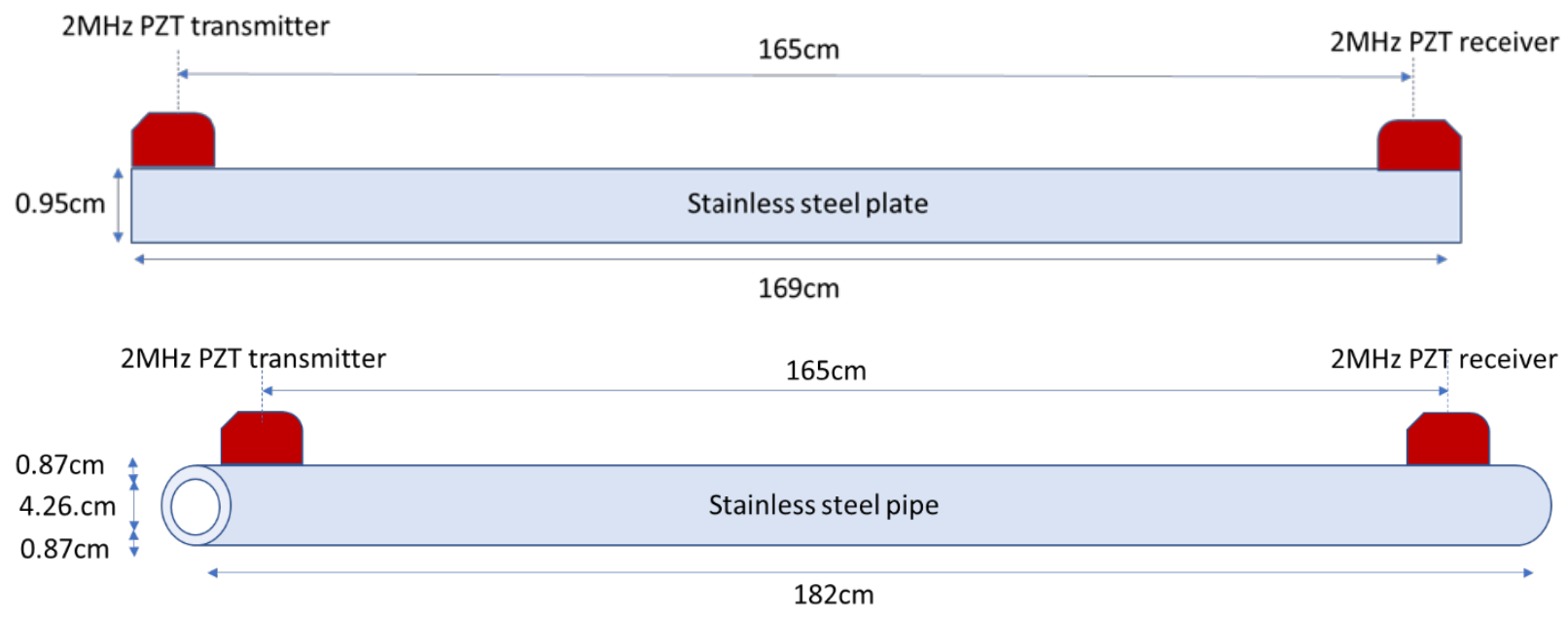

Figure 29. Communication channels based refracted shear waves in stainless steel with PZT's on $4^{\circ}$ angled wedge. The channels are stainless steel plate (top) and pipe (bottom)
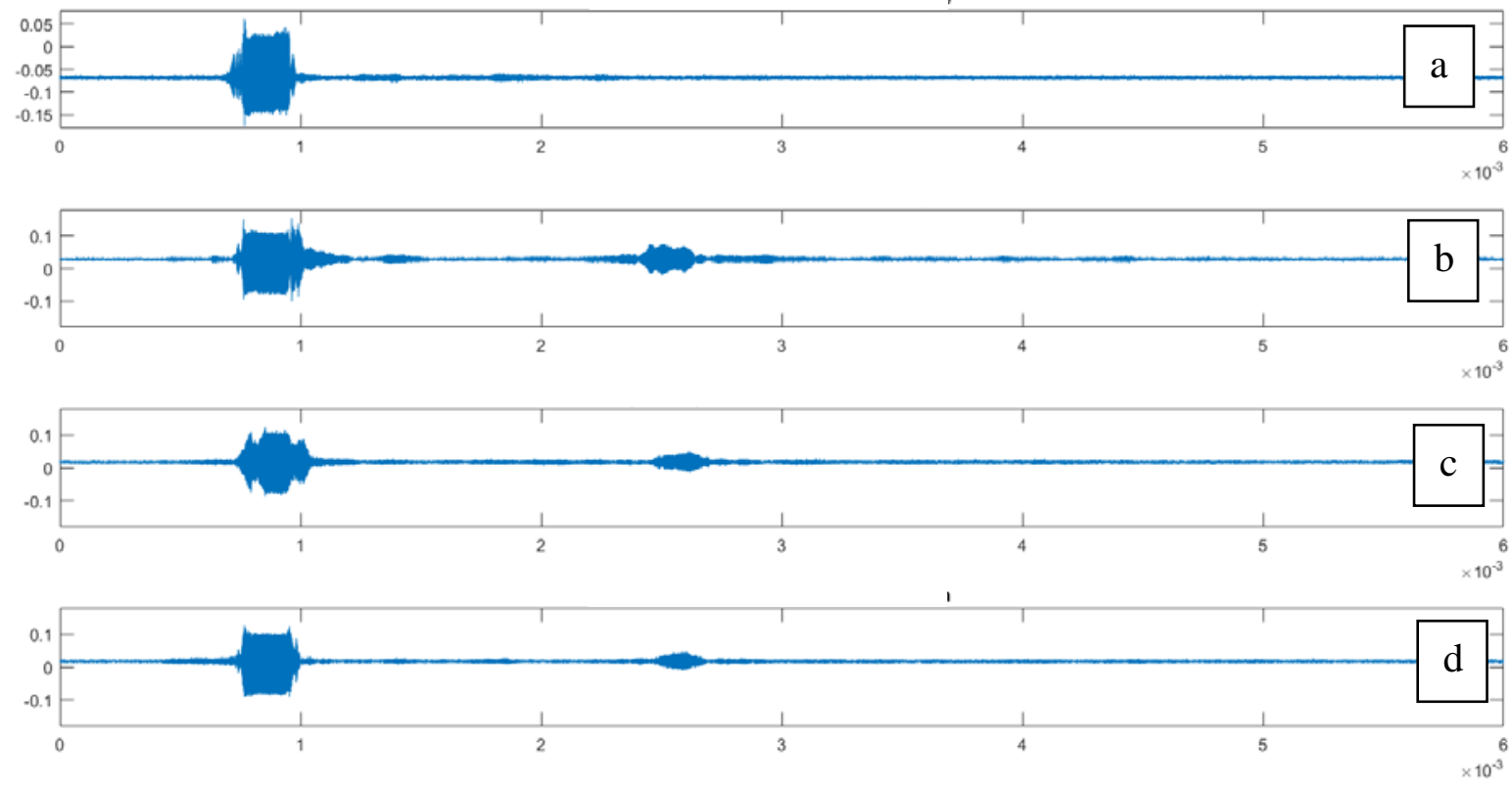

Figure 30 - Time domain signals on plate and pipe: (a) transmission on plate, (b) transmission on pipe with transmitter and receiver aligned, (c) transmission on pipe with receiver and transmitter rotated by relative $90^{\circ}$, (d) transmission on a pipe with transmitter and receiver rotated by relative $180^{\circ}$ 


\subsection{Demonstration Data Transmission with PZT-based Channel}

Demonstration image, text and sound transmission on a CVCS-type pipe with baffle plates using Red Pitaya boards and GNURadio software was performed during the Digital Environment for Advanced Reactors Workshop at ANL on June 5. In the final demonstration, information transmission with $1.8 \mathrm{MHz}$ frequency shear wave at $2 \mathrm{~KB} / \mathrm{s}$ data rate across 5 feet distance on stainless steel pipe. Figure xx show schematic of data transmission architecture and the laboratory photograph. Demonstration included transmission of an image with ANL logo (32KB of memory of computer hard drive) at $2 \mathrm{~KB} / \mathrm{s}$ data rate. Schematics of the communication system setup for transmission of images and laboratory photograph of the setup are shown in Figure 31

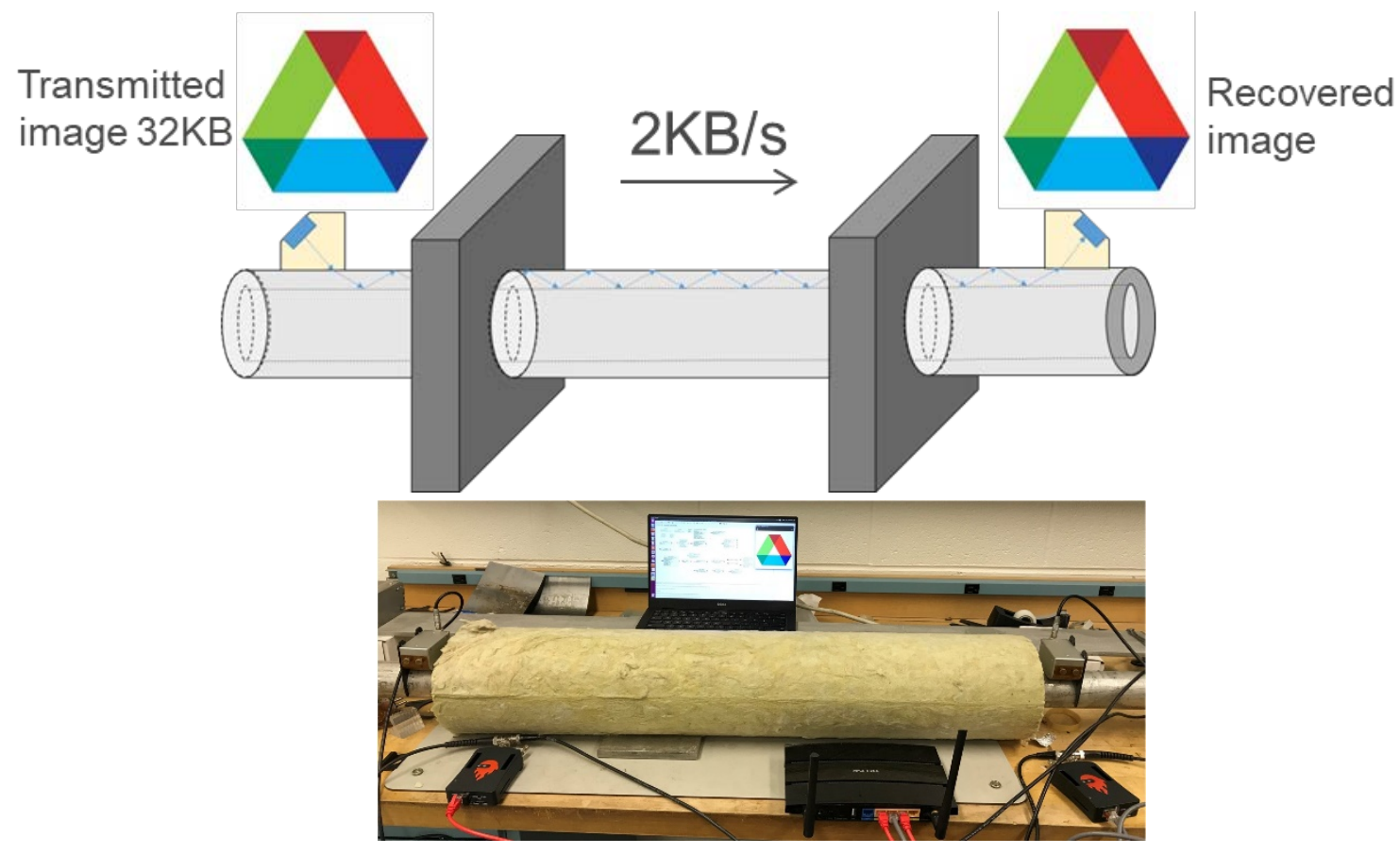

Figure 31 - Schematics of communication system setup for image transmission on a pipe (top) and laboratory photograph of the setup (bottom)

An example of text file transmitted using the same communication setup is shown in Figure 32. The left panel displays the contents of transmitted file containing the string "Hello world!" The string is continuously transmitted. The right panel of the same figure contains multiple instances of the received string. Note that no errors can be observed in repeated transmission trials. 


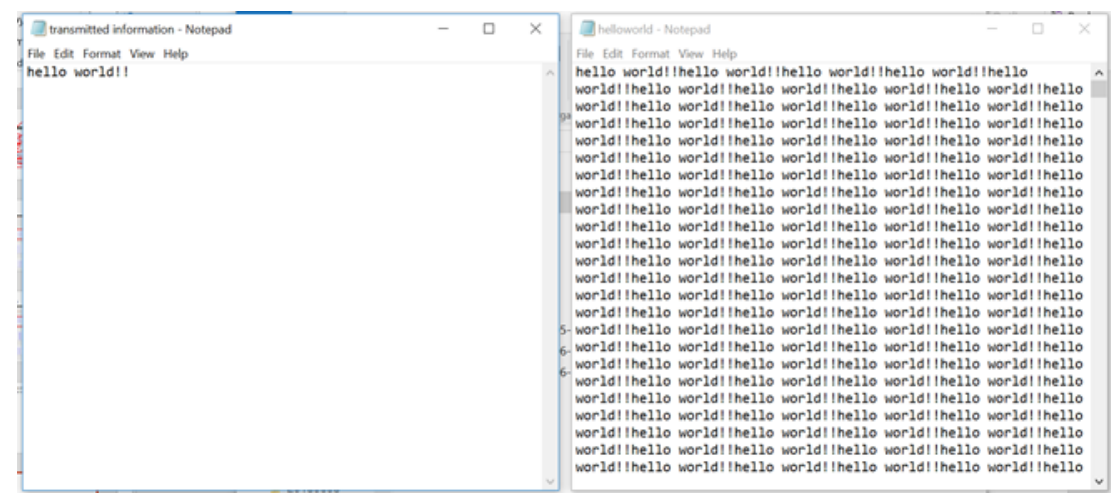

Figure 32 - Transmission of text (left) transmitted file, (b) received file

\subsection{Preliminary Evaluation of Data Transmission with EMAT-based Channel}

Shear-Horizontal (SH) wave is one of the plate waves which is well suited for ultrasonic communication. Advantages of SH wave is that they are not attenuated by water and less attenuated by the coating of the solids and it suffer little energy loss when propagate in the solids. If SH wave is obliquely incident on a free surface, there will be no mode conversion from $\mathrm{SH}$ wave to longitudinal wave or SV (shear vertical wave). Which means that the received signal is easy to analysis the multipath effect of the channel, as there is one certain type of SH wave modes exist. Generally, SH wave is generated by angle beam piezoelectric transducer and may suffer from the ambiguity in signal quality due to uncertainty in a coupling condition. However, an EMAT can selectively excite different kinds of $\mathrm{SH}$ waves requiring no direct contact with the samples. In addition, EMAT holds promise for ultrasonic signal generation in harsh environment (high temperature, radiation).

Since communication channel has finite length and EMAT transducer generate (receiver) signals in forward direction and backward direction, more reflections can be seen in the receiver side compared with PZT transducer. An experiment is setup to show the interference and reflection. The schematics of EMAT-based communication channel on a stainless-steel plate are shown in Figure 33. In order to see the see better reflection, the transmitter and receiver is set $30 \mathrm{~cm}$ from the edge.

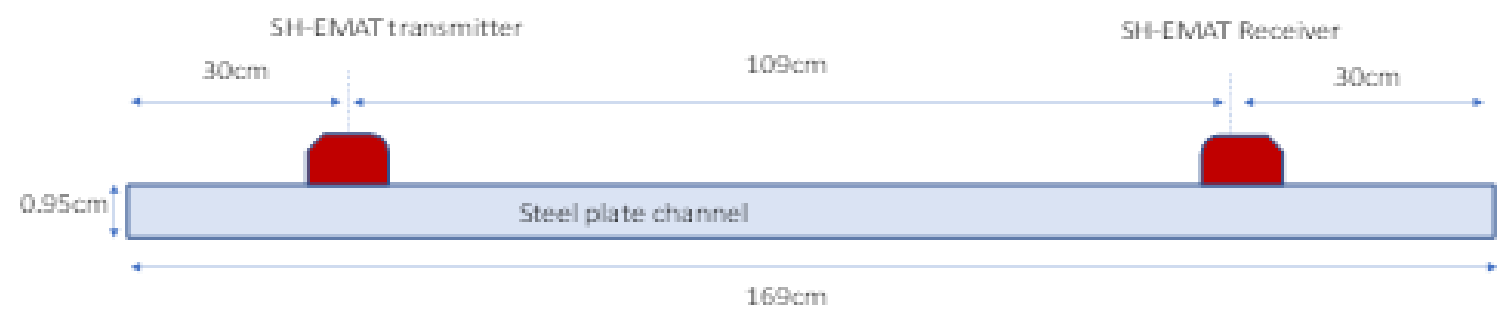

Figure 33 - Schematics of EMAT-based communication channel on a plate

A $100 \mu$ s bit duration pulse is sent by transmitter, and Figure 34 shows the signal picked up by the receiver. We can see that the amplitude of signals of different frequencies and matches with 
frequency response. The reflections from Group 1 are directly from 2 edges of steel plate and reflections from Group 2 are signals which next round reflection. SH waves are selected with different frequencies and they travel in different velocities. Group 1 signal travel 2 times long of steel plate arrives at Group 2. All these reflections will affect communication efficiency and bit error rate. We can see the attenuation is proportional frequency. It means that the lower frequency ultrasonic signal like $230 \mathrm{kHz}$ can propagation longer. However, the attenuation can restrain the Group 2 and later reflection signals. In this channel, we set transmitter and receiver next to the edge which can remove part of the group 1 reflection and set a longer bit duration to make the reflections overlap. Considering the power efficiency and less Group 2 reflection, we will choose around $400 \mathrm{kHz}$ as the carrier frequency.

\section{Group $1 \quad$ Group 2}

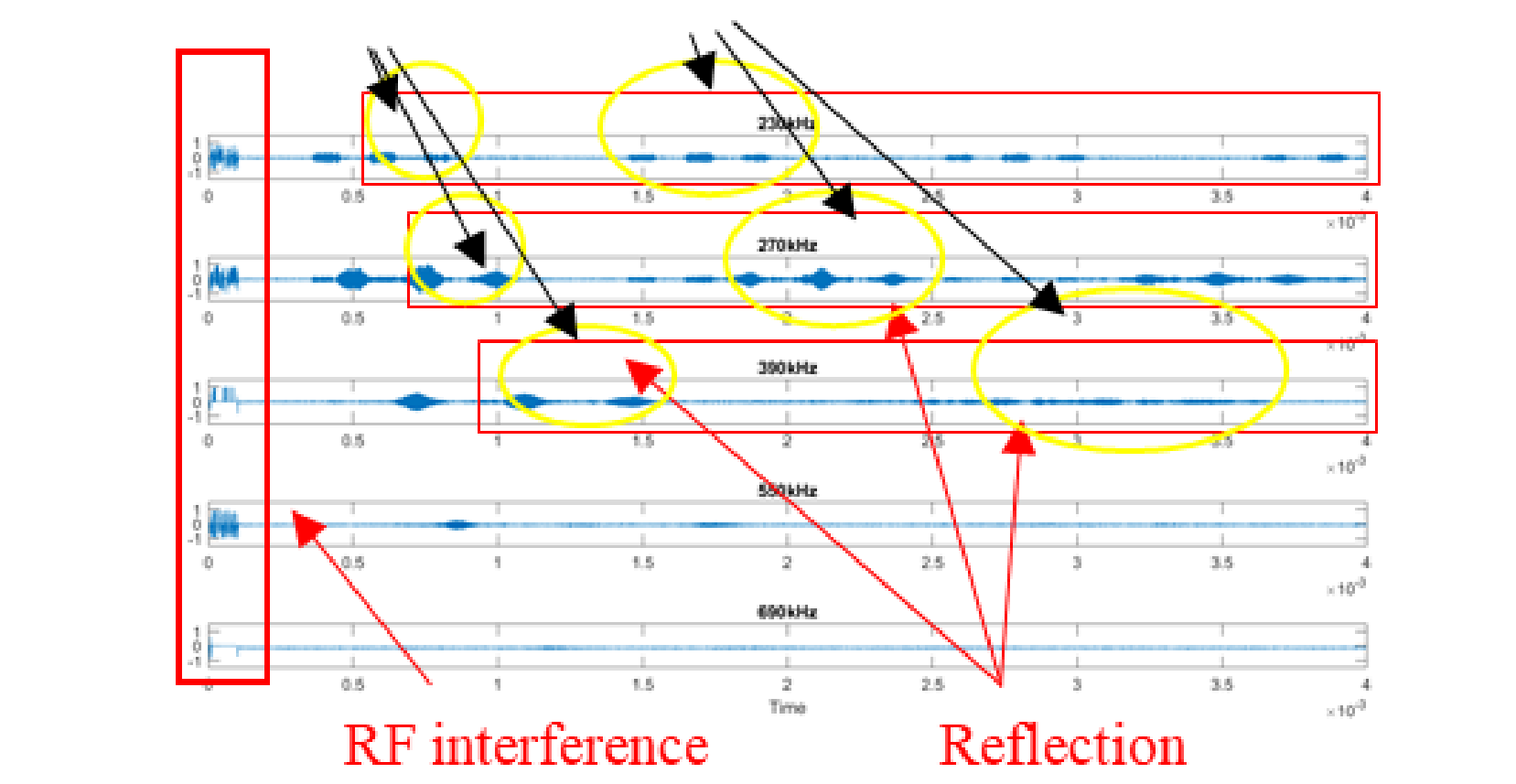

Figure 34 - Signal reflections and interferences in EMAT-based channel

EMAT transmitter needs a large power excitation to generate ultrasonic signal. Any imbalance in the communication system cause the BNC cable, power wires and every other interconnection to become part of the transmitter and radiate. the interference can be seen in Figure 34 is at least 5 times larger than the ultrasonic signal. If the interference is not removed, the system can't communicate with ultrasonic signal. As a result, an impedance matching network is implemented in receiver side to improve the quality of received signal and isolated the RF interference.

Transfer efficiency between electric energy and sound energy with an EMAT is much lower than PZT. For the transmitter side, the impedance of EMAT is similar to the power amplifier. In the receiver side, there is large impedance mismatch between EMAT receiver (140 $\Omega$ resistive) and 
low-noise amplifier (100M $\Omega$ resistive), which will cause inefficient transfer the acoustic wave into electric energy. An INNERSPEC signal conditioning box (SCB) is implemented as the impedance matching. There is ground connector on the SCB and additional ground path is added for the receiver. Meanwhile, the steel plate channel has to be grounded. The interference is highly restrained and will not affect the ultrasonic communication. 


\section{Conclusions}

This report provides recent results of software and hardware development of acoustics communication system on stainless steel pipes at a nuclear facility. Communication protocol was developed in GNURadio software defined radio (SDR) environment, with interface to Red Pitaya electronic boards. Transmission of images, text and sound was demonstrated using piezo-electric transducers (PZT) at $2 \mathrm{~KB} / \mathrm{s}$ data rate. Efforts are under way to increase data transmission rate of the communication system through algorithmic improvements in GNURadio protocol, and through optimization of hardware resources. Algorithmic improvements will include better design of automated gain controller to potentially reduce the time needed to compensate amplitude reduction of the demodulated signal. Other improvements under current study include design of decimation algorithm which tracks the rising and falling edges of the received pulse. Presently, the challenge in decimation is that the receiver is not synchronized with the transmitter. In case of hardware resource improvements, the tradeoff between transducer frequency and attenuation losses will be further investigated. In principle, losses in the channel can be compensated with low noise amplifier, so this additional constraint will be factored into design consideration. Also, attenuation in the channel could be potentially used to the benefit of communication system by suppressing echoes. Finally, custom geometry EMAT is under development to improve the efficiency of signal coupling into the pipe. 


\section{References}

Argonne National Laboratory (2018), "Realizing a Pipe Dream,” available at https://www.anl.gov/articles/realizing-pipe-dream

Bakhtiari, S, Chien, H.T., Heifetz A., Elmer, T.W (2018) "Nondestructive Testing Research and Development Efforts at Argonne National Laboratory: An Overview,” Materials Evaluation 76 (7), 911-920.

Heifetz A., Young J., Huang X., Bakhtiari S., Saniie J., Vilim R.B, (2018), “Acoustic Channel Link Models for Digital Communication Protocols,” ANL-18/25, August 15.

Heifetz A., Bakhtiari S., Huang X., Ponciroli R., and Vilim R.B. (2017) "First Annual Progress

Report on Transmission of Information by Acoustic Communication along Metal Pathways in Nuclear Facilities,” ANL/NE-17/30, September 30.

Heifetz A., Bakhtiari S., Vilim R.B. (2018) "Development of System Performance Models," Argonne National Laboratory ANL/NE-17-22.

Wang B., Saniie J., Bakhtiari S., and Heifetz A. (2017) "Architecture of an ultrasonic experimental platform for information transmission through solids," 2017 IEEE International Ultrasonics Symposium (IUS), Washington, DC, pp. 1-4. 


\section{Argonne}

Nuclear Science and Engineering (NSE) Division

Argonne National Laboratory

9700 South Cass Avenue, Bldg. 208

Argonne, IL 60439

www.anl.gov

Argonne National Laboratory is a U.S. Department of Energy laboratory managed by UChicago Argonne, LLC 